ACCEPTED 2014 July 7

Preprint typeset using LATEX style emulateapj v. 05/12/14

\title{
ON THE NATURE OF LOCAL INSTABILITIES IN ROTATING GALACTIC CORONAE AND COOL CORES OF GALAXY CLUSTERS
}

\author{
Carlo Nipoti* and Lorenzo Posti \\ Department of Physics and Astronomy, Bologna University, viale Berti-Pichat 6/2, 40127 Bologna, Italy \\ Accepted 2014 July 7
}

\begin{abstract}
A long-standing question is whether radiative cooling can lead to local condensations of cold gas in the hot atmospheres of galaxies and galaxy clusters. We address this problem by studying the nature of local instabilities in rotating, stratified, weakly magnetized, optically thin plasmas in the presence of radiative cooling and anisotropic thermal conduction. For both axisymmetric and nonaxisymmetric linear perturbations we provide the general equations that can be applied locally to specific systems to establish whether they are unstable and, in case of instability, to determine the kind of evolution (monotonically growing or over-stable) and the growth rates of unstable modes. We present results for models of rotating plasmas representative of Milky Way-like galaxy coronae and cool-cores of galaxy clusters. It is shown that the unstable modes arise from a combination of thermal, magnetothermal, magnetorotational and heat-flux-driven buoyancy instabilities. Local condensation of cold clouds tends to be hampered in cluster cool cores, while it is possible under certain conditions in rotating galactic coronae. If the magnetic field is sufficiently weak the magnetorotational instability is dominant even in these pressure supported systems.

Keywords: conduction - galaxies: clusters: intracluster medium - galaxies: ISM - instabilities magnetohydrodynamics - plasmas
\end{abstract}

\section{INTRODUCTION}

Extended hot atmospheres are believed to be ubiquitous in massive virialized systems in the Universe. These virialtemperature gaseous halos have been detected in X-rays not only in galaxy clusters (Rosati et al. 2002), but also in massive elliptical (Mathews \& Brighenti 2003) and disc (Dai et al. 2012) galaxies. A combination of different observational findings leads to the conclusion that a corona is present also in the Milky Way (Miller \& Bregman 2013). A long-standing question is whether these atmospheres are thermally unstable (in the sense of Field 1965): if local thermal instability (TI) occurs, cold gaseous clouds can condense throughout the plasma; otherwise, substantial cooling can only happen at the system center, where, however, it is expected to be opposed by feedback from the central supermassive black hole. This has important implications for the evolution of galaxy clusters (e.g. Mathews \& Bregman 1978; McCourt et al. 2012, and references therein) and galaxies (e.g. Maller \& Bullock 2004; Joung et al. 2012 , and references therein).

The evolution of thermal perturbations in astrophysical plasmas, subject to radiative cooling and thermal conduction, is a complex physical process (Field 1965), which is influenced by several factors, such as entropy stratification (Malagoli et al. 1987; Balbus \& Soker 1989; Binney et al. 2009), magnetic fields (Loewenstein 1990; Balbus 1991; Balbus \& Reynolds 2010) and rotation (Defouw 1970; Nipoti 2010, hereafter N10). In the present paper, which is the follow-up of Nipoti \& Posti (2013, hereafter NP13), we focus on the role of rotation in determining the stability properties of these plasmas, in the presence of weak magnetic fields. Rotation is clearly expected to be important in the case of the coronae of disc galaxies (Marinacci et al. 2011), but we stress that a substantial contribution of rotation could be present also in the hot gas of galaxy clusters (Bianconi et al. 2013). Though our focus is mainly on galaxy and galaxy-cluster atmospheres, it must be noted that the analysis here presented could be relevant also to the study of other rotating optically thin astrophysical plasmas, such as accretion disc coronae (Das \& Sharma 2013; Li et al. 2013).

In NP13 we have shown that rotating, radiatively cooling atmospheres with ordered weak magnetic fields (and therefore anisotropic heat conduction) are unstable to axisymmetric perturbations, in the sense that there is always at least one growing axisymmetric mode. The physical implications of this formal result clearly depend on the nature of this instability, which we try to address in the present work. In particular, we want to explore whether the linear instabilities found in NP13 are over-stabilities or monotonically growing instabilities, how the evolution of the instability depends on the properties of the perturbation and what are the driving physical mechanisms. We also extend the linear stability analysis of NP13 considering non-axisymmetric linear perturbations. We analyze the linear evolution of the instabilities in models of rotating plasmas representative of Milky Way-like galaxy coronae and cool-cores of galaxy clusters, comparing the results to those obtained for similar unmagnetized models, characterized by isotropic heat conduction. The instabilities found in the present work are interpreted in terms of well-known instabilities such as the TI, the magnetorotational instability (MRI, Balbus \& Hawley 1991; see also Velikhov 1959 and Chandrasekhar 1960), the magnetothermal instability (MTI, Balbus 2000) and the heat-flux-driven buoyancy instability (HBI, Quataert 2008).

*carlo.nipoti@unibo.it 
The paper is organized as follows. In Section 2 we present the relevant magnetohydrodynamic (MHD) equations and we define the properties of the unperturbed plasma. The results of the linear-perturbation analysis are presented in Section 3 for axisymmetric perturbations and in Section 4 for non-axisymmetric perturbations. Section 5 summarizes and concludes.

\section{GOVERNING EQUATIONS AND PROPERTIES OF THE UNPERTURBED PLASMA}

A stratified, rotating, magnetized atmosphere in the presence of thermal conduction and radiative cooling is governed by the following MHD equations:

$$
\begin{aligned}
& \frac{\partial \rho}{\partial t}+\nabla \cdot(\rho \mathbf{v})=0, \\
& \rho\left[\frac{\partial \mathbf{v}}{\partial t}+(\mathbf{v} \cdot \nabla) \mathbf{v}\right]=-\nabla\left(p+\frac{B^{2}}{8 \pi}\right)-\rho \nabla \Phi+\frac{1}{4 \pi}(\mathbf{B} \cdot \nabla) \mathbf{B}, \\
& \frac{\partial \mathbf{B}}{\partial t}-\nabla \times(\mathbf{v} \times \mathbf{B})=0, \\
& \frac{p}{\gamma-1}\left[\frac{\partial}{\partial t}+\mathbf{v} \cdot \nabla\right] \ln \left(p \rho^{-\gamma}\right)=-\nabla \cdot \mathbf{Q}-\rho \mathcal{L},
\end{aligned}
$$

supplemented by the condition that the magnetic field $\mathbf{B}$ is solenoidal $(\nabla \cdot \mathbf{B}=0)$. Here $\rho, p, T$ and $\mathbf{v}$ are, respectively, the density, pressure, temperature and velocity fields of the fluid, $\Phi$ is the external gravitational potential (we neglect self-gravity), $\gamma=5 / 3$ is the adiabatic index, $\mathbf{Q}$ is the conductive heat flux, and $\mathcal{L}=\mathcal{L}(T, \rho)$ is the radiative energy loss per unit mass of fluid. In a dilute magnetized plasma heat is significantly transported by electrons only along the magnetic field lines (see Braginskii 1965), so the conductive heat flux is given by

$$
\mathbf{Q}=-\frac{\chi \mathbf{B}(\mathbf{B} \cdot \nabla) T}{B^{2}} \quad \text { (Anisotropic conduction), }
$$

where $\chi \equiv \kappa T^{5 / 2}$ is the Spitzer electron conductivity with $\kappa \simeq 1.84 \times 10^{-5}(\ln \Lambda)^{-1} \operatorname{erg~s}^{-1} \mathrm{~cm}^{-1} \mathrm{~K}^{-7 / 2}$, and $\ln \Lambda$ is the Coulomb logarithm (Spitzer 1962). In the following we neglect the weak temperature and density dependence of ln $\Lambda$, assuming that $\kappa$ is a constant, so $\chi=\chi(T) \propto T^{5 / 2}$. We note that, even if the medium is magnetized, for simplicity we have assumed that the pressure is isotropic: in other words, we neglect the so-called Braginskii (1965) viscosity, i.e. the fact that momentum transport is anisotropic in the presence of a magnetic field. Though this approximation is not rigorously justified (see Kunz 2011; Kunz et al. 2012; Parrish et al. 2012), we adopt it just in the working hypothesis that anisotropic pressure is not the crucial factor in determining the thermal stability properties of rotating plasmas. This is a limitation of the present investigation, which must be kept in mind in the interpretation of the results.

As we consider rotating fluids, we work in cylindrical coordinates $(R, \phi, z)$, where $R=0$ is the rotation axis. The unperturbed plasma is described by time-independent axisymmetric pressure $p_{0}$, density $\rho_{0}$, temperature $T_{0}$, velocity $\mathbf{v}_{0}=\left(v_{0 R}, v_{0 \phi}, v_{0 z}\right)$ and magnetic field $\mathbf{B}_{0}=\left(B_{0 R}, B_{0 \phi}, B_{0 z}\right)$ satisfying equations (1-4) with vanishing partial derivatives with respect to $t$, under the assumption that the unperturbed magnetic field is subthermal and dynamically unimportant $\left(\beta \equiv 8 \pi p_{0} / B_{0}^{2} \gg 1\right)$. Though a stationary solution of the energy equation (4) does not necessarily imply $\rho \mathcal{L}=-\nabla \cdot \mathbf{Q}$ [for instance, in a classic stationary cooling-flow model $\rho \mathcal{L} \neq-\nabla \cdot \mathbf{Q}$ and $\mathbf{v} \cdot \nabla \ln \left(p \rho^{-\gamma}\right) \neq 0$ ], here we restrict for simplicity to the case in which cooling is balanced by heat conduction in the unperturbed system. This implies that the unperturbed magnetic field lines are not isothermal, so $\nabla T_{0} \cdot \mathbf{b}_{0} \neq 0$ in the background plasma (see Quataert 2008 for a discussion). The unperturbed fluid rotates differentially with angular velocity $\Omega(R, z) \equiv v_{0 \phi}(R, z) / R$ depending on both $R$ and $z$ (we take $\Omega \geq 0$ ). For simplicity, we assume $v_{0 R}=v_{0 z}=0$, which, combined with the assumption that all the components of the background magnetic fields are time-independent, implies that the unperturbed system satisfies Ferraro (1937) isorotation law $\mathbf{B}_{0} \cdot \nabla \Omega=0$ (see NP13 for a discussion). The unperturbed system, though magnetized, obeys the the Poincaré-Wavre theorem (Tassoul 1978), because $\beta \gg 1$, so we can distinguish barotropic $\left[p_{0}=p_{0}\left(\rho_{0}\right), \Omega=\Omega(R)\right]$ and baroclinic $\left[p_{0}=p_{0}\left(\rho_{0}, T_{0}\right), \Omega=\Omega(R, z)\right]$ distributions.

\section{AXISYMMETRIC PERTURBATIONS}

\subsection{Dispersion relation}

We linearize the governing equations (1-4) with axisymmetric Eulerian perturbations of the form $F \mathrm{e}^{-\mathrm{i} \omega t+\mathrm{i} k_{R} R+\mathrm{i} k_{z} z}$, with $|F| \ll\left|F_{0}\right|$, where $F_{0}$ is the unperturbed quantity, $\omega$ is the perturbation frequency, and $k_{R}$ and $k_{z}$ are, respectively, the radial and vertical components of the perturbation wave-vector. The linear-perturbation analysis, in the short wave-length and low frequency (Boussinesq) approximation, leads to the most general dispersion relation derived in NP13. It is convenient to express the dispersion relation as a function of $n \equiv-\mathrm{i} \omega$ : in terms of $n$, the perturbation evolves with a time dependence $F(t) \propto \mathrm{e}^{n t}$, where in general $n$ is a complex number. Therefore, stable modes are those with $\operatorname{Re}(n) \leq 0$ and unstable modes those with $\operatorname{Re}(n)>0$. Among the unstable modes we have monotonically growing unstable modes, with $\operatorname{Im}(n)=0$, and over-stable modes, with $\operatorname{Im}(n) \neq 0$, in which the perturbation oscillates with growing amplitude. The dispersion relation of NP13 (equation 32 in that paper) can be written in dimensionless 
form as

$$
\begin{aligned}
& \tilde{n}^{5}+\tilde{\omega}_{\mathrm{d}} \tilde{n}^{4}+\left[\tilde{\omega}_{\mathrm{BV}}^{2}+\tilde{\omega}_{\mathrm{rot}}^{2}+2 \tilde{\omega}_{\mathrm{A}}^{2}\right] \tilde{n}^{3}+\left[\left(\tilde{\omega}_{\mathrm{rot}}^{2}+2 \tilde{\omega}_{\mathrm{A}}^{2}\right) \tilde{\omega}_{\mathrm{d}}+\tilde{\omega}_{\mathrm{A}}^{2} \tilde{\omega}_{\mathrm{c}, \mathrm{mag}}\right] \tilde{n}^{2}+\tilde{\omega}_{\mathrm{A}}^{2}\left(\tilde{\omega}_{\mathrm{A}}^{2}+\tilde{\omega}_{\mathrm{BV}}^{2}+\tilde{\omega}_{\mathrm{rot}}^{2}-4 \frac{k_{z}^{2}}{k^{2}}+\tilde{\omega}_{\mathrm{c}, \phi}^{2}\right) \tilde{n} \\
& +\tilde{\omega}_{\mathrm{A}}^{2}\left[\left(\tilde{\omega}_{\mathrm{A}}^{2}+\tilde{\omega}_{\mathrm{rot}}^{2}-4 \frac{k_{z}^{2}}{k^{2}}\right) \tilde{\omega}_{\mathrm{d}}+\tilde{\omega}_{\mathrm{A}}^{2} \tilde{\omega}_{\mathrm{c}, \mathrm{mag}}\right]=0 \quad \text { (Magnetized). }
\end{aligned}
$$

In the above equation $\tilde{n} \equiv n / \Omega, \tilde{\omega}_{\mathrm{d}} \equiv \tilde{\omega}_{\mathrm{c}, \mathrm{a}}+\tilde{\omega}_{\mathrm{th}}\left(\right.$ where $\tilde{\omega}_{\mathrm{c}, \mathrm{a}} \equiv \omega_{\mathrm{c}, \mathrm{a}} / \Omega$ and $\left.\tilde{\omega}_{\mathrm{th}} \equiv \omega_{\mathrm{th}} / \Omega\right), \tilde{\omega}_{\mathrm{BV}}^{2} \equiv \omega_{\mathrm{BV}}^{2} / \Omega^{2}, \tilde{\omega}_{\mathrm{rot}}^{2} \equiv$ $\omega_{\mathrm{rot}}^{2} / \Omega^{2}, \tilde{\omega}_{\mathrm{A}}^{2} \equiv \omega_{\mathrm{A}}^{2} / \Omega^{2}, \tilde{\omega}_{\mathrm{c}, \mathrm{mag}} \equiv \omega_{\mathrm{c}, \mathrm{mag}} / \Omega$ and $\tilde{\omega}_{\mathrm{c}, \phi}^{2} \equiv \omega_{\mathrm{c}, \phi}^{2} / \Omega^{2}$, where

$$
\omega_{\mathrm{c}, \mathrm{a}} \equiv \frac{\left(\mathbf{k} \cdot \mathbf{b}_{0}\right)^{2}}{k^{2}} \omega_{\mathrm{c}}
$$

is the anisotropic thermal conduction frequency,

$$
\omega_{\mathrm{c}} \equiv\left(\frac{\gamma-1}{\gamma}\right) \frac{k^{2} \chi\left(T_{0}\right) T_{0}}{p_{0}}
$$

is the isotropic thermal conduction frequency,

$$
\omega_{\text {th }} \equiv-\left(\frac{\gamma-1}{\gamma}\right) \frac{\rho_{0}}{p_{0}}\left[\mathcal{L}\left(\rho_{0}, T_{0}\right)+\rho_{0} \mathcal{L}_{\rho}\left(\rho_{0}, T_{0}\right)-T_{0} \mathcal{L}_{T}\left(\rho_{0}, T_{0}\right)\right]
$$

is the thermal-instability frequency ${ }^{1}$,

$$
\omega_{\mathrm{BV}}^{2} \equiv-\frac{k_{z}^{2}}{k^{2}} \frac{\mathcal{D} p_{0}}{\rho_{0} \gamma} \mathcal{D} \ln p_{0} \rho_{0}^{-\gamma}
$$

is the Brunt-Väisälä or buoyancy frequency squared [we have introduced the differential operator $\mathcal{D} \equiv\left(k_{R} / k_{z}\right) \partial / \partial z-$ $\partial / \partial R]$

$$
\omega_{\text {rot }}^{2} \equiv-\frac{k_{z}^{2}}{k^{2}} \frac{1}{R^{3}} \mathcal{D}\left(R^{4} \Omega^{2}\right)
$$

is the angular-momentum gradient frequency squared,

$$
\omega_{\mathrm{A}}^{2} \equiv\left(\mathbf{k} \cdot \mathbf{v}_{\mathrm{A}}\right)^{2}=\frac{\left(\mathbf{k} \cdot \mathbf{B}_{0}\right)^{2}}{4 \pi \rho_{0}}
$$

is the Alfvén frequency squared, and

$$
\omega_{\mathrm{c}, \mathrm{mag}} \equiv-\omega_{\mathrm{c}} \frac{4 \pi p_{0}}{B_{0}^{2}} \frac{k_{z}^{2}}{k^{2}} \frac{\mathcal{D} \ln p_{0}}{k^{2}}\left[\mathcal{D} \ln T_{0}-2\left(\nabla \ln T_{0} \cdot \mathbf{b}_{0}\right)\left(\frac{k_{R}}{k_{z}} b_{0 z}-b_{0 R}\right)\right]
$$

and

$$
\omega_{\mathrm{c}, \phi}^{2} \equiv \omega_{\mathrm{c}} \Omega \frac{8 \pi p_{0}}{B_{0}^{2}} \frac{k_{z}^{2}}{k^{2}} \frac{\mathcal{D} \ln p_{0}}{k^{2}}\left(\nabla \ln T_{0} \cdot \mathbf{b}_{0}\right) b_{0 \phi}
$$

are other two frequencies associated to thermal conduction mediated by the magnetic field.

We are interested in comparing the thermal stability properties of plasmas with ordered magnetic field to those of unmagnetized fluids with similar properties, in which thermal conduction is isotropic. In this case the governing equations are the usual hydrodynamic equations, which can be obtained from equations (1), (2) and (4), imposing $\mathbf{B}=0$ and conductive heat flux (Spitzer 1962)

$$
\mathbf{Q}=-f \kappa T^{5 / 2} \nabla T, \quad \text { (Isotropic conduction), }
$$

where we allow for the possibility that thermal conduction is suppressed to a fraction $f$ of the classical Spitzer's value (Binney \& Cowie 1981), so that the unmagnetized case can also represent a simple model of system with tangled magnetic fields. The dispersion relation is the one derived in N10 (equation 16 in that paper), which we write here as a function of $\tilde{n}=n / \Omega$ :

$$
\tilde{n}^{3}+\tilde{n}^{2} \tilde{\omega}_{\mathrm{d}}+\left(\tilde{\omega}_{\mathrm{BV}}^{2}+\tilde{\omega}_{\mathrm{rot}}^{2}\right) \tilde{n}+\tilde{\omega}_{\mathrm{rot}}^{2} \tilde{\omega}_{\mathrm{d}}=0 \quad \text { (Unmagnetized) }
$$

where now $\tilde{\omega}_{\mathrm{d}} \equiv f \tilde{\omega}_{\mathrm{c}}+\tilde{\omega}_{\mathrm{th}}$, with $\tilde{\omega}_{\mathrm{c}} \equiv \omega_{\mathrm{c}} / \Omega$.

1 This is a general definition of $\omega_{\mathrm{th}}$, which applies also in the case in which $\rho \mathcal{L} \neq-\nabla \cdot \mathbf{Q}$ in the unperturbed system: it can be shown that the first term in square brackets is negligible if cooling is balanced by heat conduction in the background fluid. 
Table 1

\begin{tabular}{|c|c|c|c|c|c|c|c|c|c|c|c|c|c|c|}
\hline Name & $\Gamma_{\Omega R}$ & $\Gamma_{\Omega z}$ & $\Gamma_{p R}$ & $\Gamma_{p z}$ & $\Gamma_{T R}$ & $\tilde{c}_{0}$ & $\tilde{\omega}_{\text {th }}$ & $\tilde{\omega}_{\mathrm{c}, 0}$ & $\beta$ & $b_{0 \phi}$ & $b_{0 R}$ & $b_{0 z}$ & $\overline{\Gamma_{T z}}$ & $\Gamma_{T p}$ \\
\hline MWG-bt & -0.944 & 0 & -0.321 & -0.565 & -0.042 & 1.238 & -0.052 & 0.010 & 29.15 & 0 & 0 & 1.000 & -0.074 & 0.130 \\
\hline & 44 & -0.1 & -0.321 & -0.565 & -0.0 & 1.238 & -0 & & 29.15 & 0 & -0 . & 89 & 0.503 & \\
\hline $\mathrm{CCC}$ & 368 & 0 & -0.249 & -0.324 & 0.1 & 1.834 & -0 & 28 & 73 & 0 & 0 & 00 & 0.225 & -0.695 \\
\hline CCC-bc & -0.868 & -0.130 & -0.249 & -0.324 & 0.173 & 1.834 & -0.061 & 0.128 & 77.73 & 0 & -0.148 & 0.989 & 0.537 & \\
\hline
\end{tabular}

Note. - List of dimensionless parameters of the models (see Section 3.2). The first ten parameters are independent: logarithmic slopes of the radial $\left(\Gamma_{\Omega R}\right)$ and vertical $\left(\Gamma_{\Omega z}\right)$ angular velocity gradients, of the radial $\left(\Gamma_{p R}\right)$ and vertical $\left(\Gamma_{p z}\right)$ pressure gradients, and of the radial temperature gradient $\left(\Gamma_{T R}\right)$, normalized sound speed $\left(\tilde{c}_{0}\right)$, normalized thermal-instability $\left(\tilde{\omega}_{\text {th }}\right)$ and thermal-conduction $\left(\tilde{\omega}_{\mathrm{c}, 0}\right)$ frequencies, thermal to magnetic pressure ratio $(\beta)$, and normalized azimuthal magnetic field component $\left(b_{0 \phi}\right)$. The last four parameters depend on the first ten: normalized radial $\left(b_{0 R}\right)$ and vertical $\left(b_{0 z}\right)$ magnetic field components, logarithmic slope of the vertical temperature gradient $\left(\Gamma_{T z}\right)$ and logarithmic slope of the temperature variation with respect to pressure $\left(\Gamma_{T p}\right.$; defined only for barotropic distributions).

\subsection{Parameters}

We show here that the coefficients of the dispersion relation (6) are fully defined if the values of the axisymmetric perturbation wave-vector $\mathbf{k}=\left(k_{R}, 0, k_{z}\right)$ and of ten dimensionless parameters (depending on the physical properties of the unperturbed plasma) are specified. For this purpose, it is convenient to rewrite the characteristic frequencies appearing in the coefficients as follows:

$$
\begin{aligned}
& \tilde{\omega}_{\mathrm{c}, \mathrm{a}}=\frac{k^{2} R^{2}}{1+x^{2}}\left(b_{0 z}+x b_{0 R}\right)^{2} \tilde{\omega}_{\mathrm{c}, 0} \\
& \tilde{\omega}_{\mathrm{c}}=k^{2} R^{2} \tilde{\omega}_{\mathrm{c}, 0} \\
& \tilde{\omega}_{\mathrm{c}, \mathrm{mag}}=-\frac{\beta}{2\left(1+x^{2}\right)} \tilde{\omega}_{\mathrm{c}, 0}\left(x \Gamma_{p z}-\Gamma_{p R}\right)\left[\left(x \Gamma_{T z}-\Gamma_{T R}\right)-2\left(\Gamma_{T R} b_{0 R}+\Gamma_{T z} b_{0 z}\right)\left(x b_{0 z}-b_{0 R}\right)\right], \\
& \tilde{\omega}_{\mathrm{c}, \phi}^{2}=\frac{\beta}{1+x^{2}} \tilde{\omega}_{\mathrm{c}, 0}\left(x \Gamma_{p z}-\Gamma_{p R}\right)\left(\Gamma_{T R} b_{0 R}+\Gamma_{T z} b_{0 z}\right) b_{0 \phi} \\
& \tilde{\omega}_{\mathrm{BV}}^{2}=-\frac{\tilde{c}_{0}^{2}}{1+x^{2}}\left(x \Gamma_{p z}-\Gamma_{p R}\right)\left[x \Gamma_{T z}-\Gamma_{T R}-\frac{\gamma-1}{\gamma}\left(x \Gamma_{p z}-\Gamma_{p R}\right)\right] \\
& \tilde{\omega}_{\mathrm{rot}}^{2}=\frac{2}{1+x^{2}}\left(\Gamma_{\Omega R}+2-x \Gamma_{\Omega z}\right) \\
& \tilde{\omega}_{\mathrm{A}}^{2}=\frac{2 \tilde{c}_{0}^{2}}{\beta} \frac{k^{2} R^{2}}{1+x^{2}}\left(x b_{0 R}+b_{0 z}\right)^{2} \\
& \tilde{\omega}_{\mathrm{th}} \equiv-\frac{R}{v_{0 \phi}}\left(\frac{\gamma-1}{\gamma}\right) \frac{\rho_{0}}{p_{0}}\left[\mathcal{L}\left(\rho_{0}, T_{0}\right)+\rho_{0} \mathcal{L}_{\rho}\left(\rho_{0}, T_{0}\right)-T_{0} \mathcal{L}_{T}\left(\rho_{0}, T_{0}\right)\right]
\end{aligned}
$$

where we have introduced the dimensionless parameters $x \equiv k_{R} / k_{z}, \mathbf{b}_{0} \equiv \mathbf{B}_{0} / B_{0}, \tilde{c}_{0} \equiv c_{0} / v_{0 \phi}\left(c_{0}\right.$ is the isothermal sound speed),

$$
\begin{aligned}
\Gamma_{p R} \equiv \frac{\partial \ln p_{0}}{\partial \ln R}, & \Gamma_{p z} \equiv \frac{R}{z} \frac{\partial \ln p_{0}}{\partial \ln |z|}, \\
\Gamma_{T R} \equiv \frac{\partial \ln T_{0}}{\partial \ln R}, & \Gamma_{T z} \equiv \frac{R}{z} \frac{\partial \ln T_{0}}{\partial \ln |z|}, \\
\Gamma_{\Omega R} \equiv \frac{\partial \ln \Omega}{\partial \ln R}, & \Gamma_{\Omega z} \equiv \frac{R}{z} \frac{\partial \ln \Omega}{\partial \ln |z|}
\end{aligned}
$$

and

$$
\tilde{\omega}_{\mathrm{c}, 0} \equiv \frac{\gamma-1}{\gamma} \frac{\chi\left(T_{0}\right) T_{0}}{v_{0 \phi} R p_{0}} .
$$

In summary, for given $\mathbf{k}$, the dispersion relation (6) for a magnetized plasma is completely defined by the choice of the following ten free parameters, depending only on the properties of the unperturbed plasma: $\Gamma_{\Omega R}$ and $\Gamma_{\Omega z}(\operatorname{logarithmic}$ slopes of the radial and vertical angular velocity gradients), $\Gamma_{p R}$ and $\Gamma_{p z}$ (logarithmic slopes of the radial and vertical pressure gradients), $\Gamma_{T R}$ (logarithmic slope of the radial temperature gradient), $\tilde{c}_{0}$ (sound to rotation speed ratio), $\tilde{\omega}_{\text {th }}$ and $\tilde{\omega}_{\mathrm{c}, 0}$ (normalized thermal-instability and thermal-conduction frequencies), $\beta$ (thermal to magnetic pressure ratio) and $b_{0 \phi}$ (normalized azimuthal magnetic field component). The other three parameters appearing in the definitions of the coefficients of the dispersion relations $\left(b_{0 R}, b_{0 \phi}\right.$ and $\left.\Gamma_{T z}\right)$ are not independent of the first ten listed above: $b_{0 R}$ 
Table 2

\begin{tabular}{lllllll}
\hline Name & $T_{0} / K$ & $n_{e, 0} / \mathrm{cm}^{-3}$ & $R / \mathrm{kpc}$ & $v_{0 \phi} / \mathrm{km} \mathrm{s}^{-1}$ & $Z / Z_{\odot}$ & $B_{0} / \mu G$ \\
\hline MWG & $2.15 \times 10^{6}$ & $8.58 \times 10^{-4}$ & 16.92 & 140.2 & 0.03 & 0.5 \\
CCC & $7.96 \times 10^{7}$ & $2.47 \times 10^{-2}$ & 92.00 & 576.0 & 0.30 & 10 \\
\hline
\end{tabular}

Note. - Examples of combinations of physical parameters (see Section 3.3) giving $\tilde{c}_{0}, \tilde{\omega}_{\mathrm{th}}, \tilde{\omega}_{\mathrm{c}, 0}$ and $\beta$ as in Table 1 . T T is the gas temperature, $n_{e, 0}$ is the electron number density, $R$ is the local radial coordinate (representing the characteristic physical size of the system), $Z$ is the metallicity and $B_{0}$ is the magnetic field modulus. Here we estimate $\mathcal{L}\left(\rho_{0}, T_{0}\right)$, appearing in $\tilde{\omega}_{\text {th }}$, using the collisional ionization equilibrium cooling function of Sutherland \& Dopita (1993).

and $b_{0 z}$ are determined (modulo the sign) when $\Gamma_{\Omega z}, \Gamma_{\Omega R}$ and $b_{0 \phi}$ are given, because $\Gamma_{\Omega z} b_{0 z}=-\Gamma_{\Omega R} b_{0 R}$ (isorotation condition) and $b_{0 R}^{2}+b_{0 z}^{2}+b_{0 \phi}^{2}=1 ; \Gamma_{T z}$ is determined by the vorticity equation (derived from the momentum equations), which can be written in dimensionless form as

$$
\Gamma_{\Omega z}=\frac{\tilde{c}_{0}^{2}}{2}\left(\Gamma_{T z} \Gamma_{p R}-\Gamma_{T R} \Gamma_{p z}\right) .
$$

In the special case of barotropic distributions $\Gamma_{T R}=\Gamma_{T p} \Gamma_{p R}$ and $\Gamma_{T z}=\Gamma_{T p} \Gamma_{p z}$, where

$$
\Gamma_{T p} \equiv \frac{\mathrm{d} \ln T_{0}}{\mathrm{~d} \ln p_{0}}
$$

so $\Gamma_{\Omega z}=0$ (see equation 29) and $b_{0 R}=0$ (and therefore $b_{0 z}^{2}+b_{0 \phi}^{2}=1$ ), because of the isorotation condition $b_{0 R} \Gamma_{\Omega R}=0$. We note that, at fixed $\Gamma_{T R}, \Gamma_{T z}$ and $\mathbf{b}_{0}$, the requirement that in the unperturbed system cooling is balanced by heat conduction $(\rho \mathcal{L}=-\nabla \cdot \mathbf{Q})$ can be fulfilled, for given $\rho \mathcal{L}$, by fixing the values of the magnetic field gradient and of the second spatial derivatives of the temperature. The dispersion relation (16) for an unmagnetized fluid is fully characterized by the parameters $\Gamma_{\Omega R}, \Gamma_{\Omega z}, \Gamma_{p R}, \Gamma_{p z}, \Gamma_{T R}, \tilde{c}_{0}, \tilde{\omega}_{\mathrm{th}}, \tilde{\omega}_{\mathrm{c}, 0}$ and $f \leq 1$.

\subsection{Model astrophysical plasmas}

To study the linear evolution of given modes, we must specify the numerical values of the coefficients of the dispersion relations (6) and (16). Given the number of parameters (see Section 3.2), it is clear that an exploration of parameter space is prohibitive. However, it is useful to present results for a few illustrative cases. We focus on two reference sets of models, which are meant to be representative of the physical conditions in the cool core of a massive galaxy cluster (set of models CCC) and in the corona of a Milky Way-like galaxy (set of models MWG). In all cases the hot gas is assumed to be rotating differentially, and we consider both barotropic $\left[\Omega=\Omega(R)\right.$, so $\Gamma_{\Omega z}=0$; models CCC-bt and MWG-bt $]$ and baroclinic $\left[\Omega=\Omega(R, z)\right.$, so $\Gamma_{\Omega z} \neq 0$; models CCC-bc and MWG-bt] distributions. In these models the magnetic field has no azimuthal component $\left(b_{0 \phi}=0\right)$, but we also present results for two models with $b_{0 \phi} \neq 0$ (models MWG-bt-az and MWG-bc-az). Physically, the main differences between the two sets are that temperature is higher (i.e. conduction more efficient) and rotation less important in models CCC than in models MWG, and that the radial temperature gradient is positive in models CCC and negative in models MWG. The values of the dimensionless parameters for all the models are reported in Table 1. For each of the above models we construct a corresponding unmagnetized model that has the same values of the parameters $\Gamma_{\Omega R}, \Gamma_{\Omega z}, \Gamma_{p R}, \Gamma_{p z}, \Gamma_{T R}, \tilde{c}_{0}, \tilde{\omega}_{\mathrm{th}}$ and $\tilde{\omega}_{\mathrm{c}, 0}$.

Some of the parameters listed in Table 1 are purely "geometrical": those indicated with $\Gamma$ describe the local density, pressure, temperature and velocity gradients, while $\mathbf{b}$ describes the geometry of the local magnetic field. The others $\left(\tilde{c}_{0}\right.$, $\left.\tilde{\omega}_{\mathrm{th}}, \tilde{\omega}_{\mathrm{c}, 0}, \beta\right)$ are combinations of physical parameters of the unperturbed system, but clearly they are not associated to specific values of the parameters in physical units. For instance, a given numerical value of $\tilde{c}_{0}=c_{0} / v_{0 \phi}$ can be obtained with an arbitrary value of the sound speed $c_{0}$ (in physical units), provided that the rotation speed $v_{0 \phi}$ is opportunely rescaled. In this sense, our models are not univocally associated to given physical scales, temperatures, densities, velocities and magnetic fields. Nevertheless, for illustrative purposes, it is useful to give examples of combinations of physical parameters (relevant to astrophysical applications) that produce the values of the dimensionless parameters reported in Table 1 . The quantities entering the definition of $\tilde{c}_{0}, \tilde{\omega}_{\mathrm{th}}, \tilde{\omega}_{\mathrm{c}, 0}$ and $\beta$ are the local temperature $T_{0}$, the local density $\rho_{0}$ (or, alternatively, the local electron number density $n_{e, 0}$ ), the local radial coordinate $R$ (representing the characteristic physical size of the system), the local rotation speed $v_{0 \phi}$, the metallicity $Z$ (because of the dependence of $\mathcal{L}$, an therefore of $\tilde{\omega}_{\text {th }}$, on $Z$ ) and the modulus of the local magnetic field $B_{0}$. Two examples of combinations of the values of these parameters are reported in Table 2: it is clear that model CCC can be interpreted as the cool core of a massive cluster of galaxies and model MWG as a galactic corona. In fact, the values of the dimensionless parameters of our sets of models MWG and CCC were inspired, respectively, by the Milky Way-like corona models of Binney et al. (2009) and by the cool-core cluster models of Bianconi et al. (2013). In global models of hot atmospheres (such as those of Binney et al. 2009 and Bianconi et al. 2013) the physical parameters (and then the detailed stability properties) are clearly position-dependent. In the present work we do not explore global models, but we just take specific values of the parameters that are representative of the physical conditions in typical galaxies or clusters. However, we stress that once a global model of a rotating atmosphere is given (either analytic or obtained with a numerical simulation), its thermal stability can be studied at any point of the system by solving the dispersion relations (6) and (16) with the values of the coefficients given by the physical properties at that point. 



Figure 1. Domains of stability (white), monotonic instability (dark grey) and over-stability (light grey) in the plane $k_{R} / k_{z}$ (ratio of radial and vertical wave-vector components) vs $k$ (wave-number) for barotropic (left-hand panel) and baroclinic (right-hand panel) Milky-Way like galaxy models with with $b_{0 \phi}=0$ (see Table 1). Representative contours of constant growth rate (labeled in units of $10^{-3} \Omega^{-1}$ ) are shown for the monotonically unstable modes. In both panels the dominant instabilities are due to a combination of magnetothermal and thermal modes (label MTI+TI). Here $k$ is in units of $R^{-1}$.
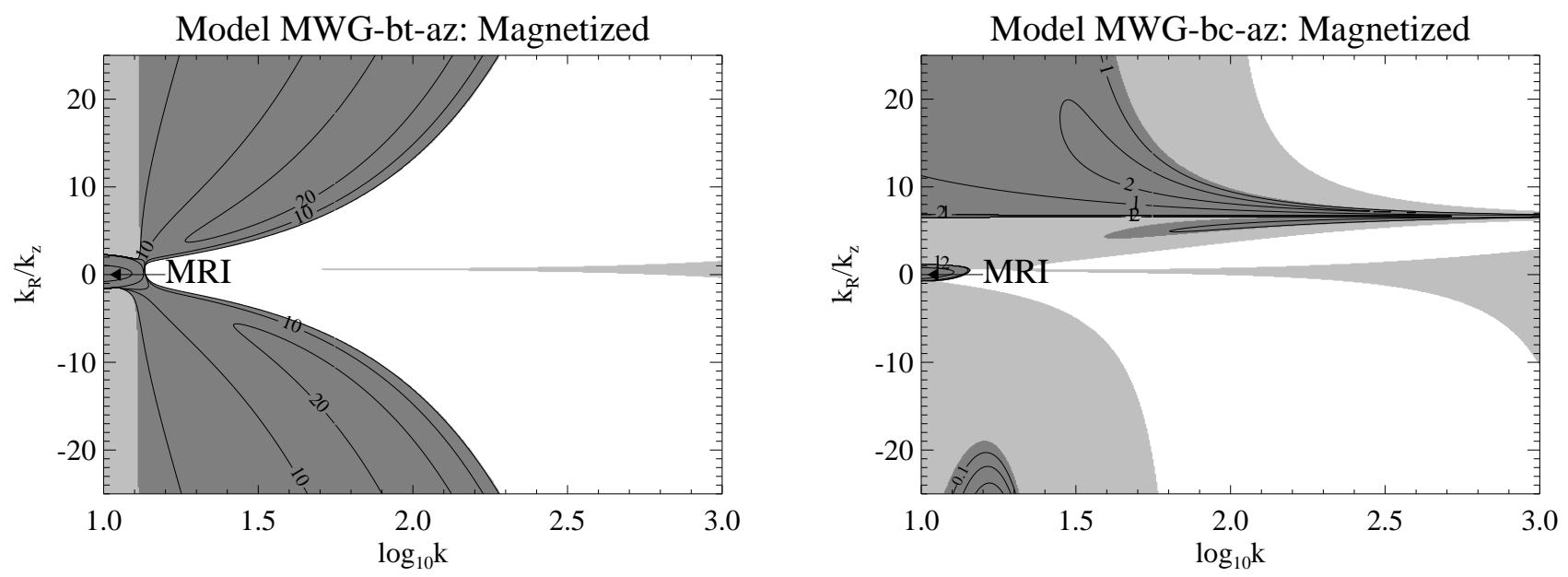

Figure 2. Same as Fig. 1, but for barotropic (left-hand panel) and baroclinic (right-hand panel) Milky-Way like galaxy models with $b_{0 \phi} \neq 0$ (see Table 1). In both panels the fastest growing monotonic instabilities (growth rate up to $\approx 440$ in units of $10^{-3} \Omega^{-1}$ ) are magnetorotational modes with $\left|k_{R} / k_{z}\right| \lesssim 1$ and $\log _{10} k \lesssim 1.1$ (label MRI).

\subsection{Results for axisymmetric perturbations}

Given a plasma model, we solved numerically the dispersion relations (6) and (16) for a large number of axisymmetric wave-vectors, using the IDL (Interactive Data Language) routine FZ_ROOTS. The analysis of the roots as functions of the wave-vector components allows us to identify domains of stability, over-stability and monotonic instability. In order to understand the nature of the instabilities it is important to assess the role of different physical mechanisms, such as radiative cooling, rotation and anisotropic heat conduction. This task is by no means easy, because the different modes are entangled and the high order of the dispersion relation makes it difficult to isolate the various contributions. Nevertheless, inspecting the behavior and the growth rates of the individual branches of the solutions as functions of the wave-number and comparing the results with those obtained for simpler configurations (for instance in the absence of radiative cooling, rotation or magnetic field), we were able to identify the different branches in terms of combinations of well known modes (TI, MRI, MTI, HBI, rotation, buoyancy and Alfvén modes).

\subsubsection{Domains of stability, over-stability and monotonically growing instability}

Here we discuss the domains, in wave-vector space, of stability, over-stability and monotonic instability against axisymmetric perturbations for the plasma models described in Section 3.3. The results for the Milky-Way like galaxy models MWG-bt (barotropic) and MWG-bc (baroclinic) with $b_{0 \phi}=0$ are shown in Fig. 1: in these plots the stability domains are represented in the space $k-x$, where $k$ is the wave-number of the perturbation and $x \equiv k_{R} / k_{z}$ is the ratio of the radial and vertical components of the wave-vector. In particular, we sampled the intervals $10 \leq k R \leq 1000$ 

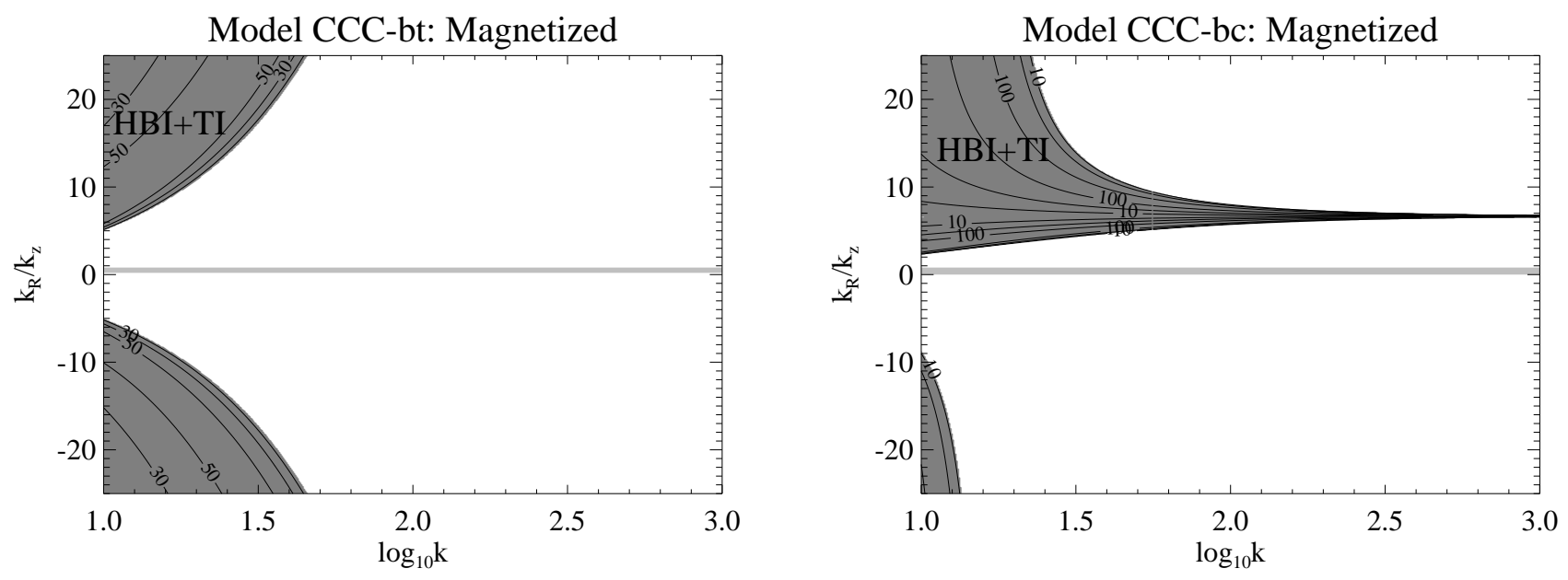

Figure 3. Same as Fig. 1, but for barotropic (left-hand panel) and baroclinic (right-hand panel) cool-core cluster models (see Table 1). In both panels the dominant instabilities are due to a combination of heat-flux driven buoyancy and thermal modes (label HBI+TI).

and $-25 \leq x \leq 25$. From Fig. 1 it is apparent that in all cases there are regions in the space $k$ - $x$ corresponding to monotonically unstable and over-stable modes, typically for small $k$ (i.e., long wave-length) and large $|x|$ (i.e. almost vertical wave-crests). In the plot representative contours of constant growth rate are shown for the monotonically unstable modes, which in this case are due to a combination of the MTI and the TI (see Section 3.4.2). In the baroclinic MWG model (right-hand panel in Fig. 1) monotonic instability occurs also at short wave-lengths (a region of stability or over-stability for barotropic models) for $x \approx-b_{0 z} / b_{0 R} \simeq 6.7$ : physically this is due to the fact that for those modes the projection of the magnetic field onto the wave vector is close to zero, so the fluid basically behaves as if it were unmagnetized $\left(\omega_{\mathrm{A}} \approx 0\right)$ and with no heat conduction $\left(\omega_{\mathrm{c}, \mathrm{a}} \approx 0\right.$; see also NP13). The driving term of these growing disturbances is $\omega_{\text {th }}$, so the instability is essentially a TI (see Section 3.4.2). The domains of stability and instability in models with $b_{0 \phi} \neq 0$ (Fig. 2) present a region of instability for low $k$ and $|x| \lesssim 1$, which is not present in the corresponding models with $b_{0 \phi}=0$ (Fig. 1): we will show in Section 3.4.2 that these unstable modes are dominated by the MRI.

The stability and instability domains of the barotropic (CCC-bt) and baroclinic (CCC-bc) cluster cool core models are qualitatively similar to those of corresponding MWG models, as it is apparent by comparing Fig. 3 with Fig. 1. Quantitatively, in the $k$-x space, the regions of monotonic instability are less extended in the CCC models than in the MWG models, mainly because damping by thermal conduction is more effective in the higher-temperature plasma of cool cores than in galactic coronae. Moreover, as we will discuss in Section 3.4.2, the driving instability is different in the two cases (HBI in models CCC, MTI in models MWG).

It is interesting to compare the above results for magnetized plasma models to those obtained for the corresponding unmagnetized models (see Section 3.3), for which the stability domains can be expressed analytically in terms of the quantities $\tilde{\omega}_{\mathrm{d}}, \tilde{\omega}_{\text {rot }}^{2}$ and $\tilde{\omega}_{\mathrm{BV}}^{2}$ (see N10, figure 1 in that paper). The behavior of the barotropic models with no magnetic field is very simple: all modes with $k>k_{\min }$ (i.e. sufficiently short wave-length perturbations) are either stable or over-stable (due to effective heat-conduction damping), while for $k<k_{\min }$ we have monotonic instability: for instance, for model MWG-bt $k_{\min } \simeq 22(f / 0.01)^{-1 / 2}$, where $f \leq 1$ is the thermal-conduction suppression factor ${ }^{2}$. Baroclinic models are characterized also by a critical wave-vector component ratio $x_{\text {crit }}$ (for instance, $x_{\text {crit }} \simeq-7$ for model MWG$\mathrm{bc}$ ): for $x>x_{\text {crit }}$ the behavior is similar to that of the corresponding barotropic models, while for $x<x_{\text {crit }}$ the system is rotationally unstable $\left(\omega_{\text {rot }}^{2}<0\right)$, which, combined with the Field criterion, gives over-stability for $k<k_{\text {min }}$ and instability for $k>k_{\min }($ see $\mathrm{N} 10)$.

\subsubsection{Physical nature and growth rates of the unstable modes}

In order to understand the astrophysical implications of the instabilities appearing in Figs. 1-3 it is important to determine their physical nature. In particular, an interesting question is whether the instabilities lead to local condensation and formation of cold clouds (i.e. if they are TIs in the sense of Field 1965). Clearly, the unstable modes here considered arise from a combination of different physical mechanisms. In fact, as discussed in NP13, even in the absence of radiative cooling the considered systems are exposed to other instabilities such as the MRI, the MTI and the HBI. It is useful to look at the behavior of the roots of the dispersion relations (6) and (16) in more detail: in particular, here we discuss how the different branches of the solutions depend on the wave-number $k$ at fixed radial to vertical wave-vector component ratio $x=k_{R} / k_{z}$. Though several modes (TI, MTI, HBI, MRI, buoyancy, rotation, Alfvén waves) are at work, for most branches it is possible to identify the dominant modes. For instance, in Fig. 4 we show the results obtained for the barotropic models MWG-bt and CCC-bt fixing $x=10$. The oscillating branches (imaginary parts) are neatly identified with buoyancy (Brunt-Väisälä) and Alfvén modes. Focusing on the

\footnotetext{
${ }^{2}$ In the unmagnetized case, the condition for heat-conduction damping is $\tilde{\omega}_{\mathrm{d}}=f \tilde{\omega}_{\mathrm{c}}+\tilde{\omega}_{\mathrm{th}} \geq 0$, where, for fixed unperturbed physical parameters, $\tilde{\omega}_{\mathrm{c}} \propto k^{2}$ and $\tilde{\omega}_{\mathrm{th}}=$ const. The minimum stable wave-number $k_{\min }$ corresponds to $\tilde{\tilde{\omega}}_{\mathrm{d}}=0$, so $k_{\min }^{2} f=$ const.
} 



Figure 4. Real and imaginary parts of the roots of the dispersion relation (6) as functions of the wave-number $k$ for models MWG-bt (left-hand panel) and CCC-bt (right-hand panel) when $k_{R} / k_{z}=10$. Here $k$ is in units of $R^{-1}$. For the sake of clarity here we extend the range in $k$ down to $k R=1$, but we recall that the results of the linear analysis are rigorous only for $k R \gg 1$. Unstable modes are those with $\operatorname{Re}(n)>0$ : monotonically growing if $\operatorname{Im}(n)=0$, over-stable if $\operatorname{Im}(n) \neq 0$. We identify the different unstable branches as combinations of thermal instability (TI), heat-flux driven buoyancy instability (HBI), magneto-thermal instability (MTI), buoyancy and rotation. In the imaginary plane we identify the branches in terms of Brunt-Väisälä (buoyancy) and Alfvén modes. Each root is represented with the same color in the upper and lower panels.
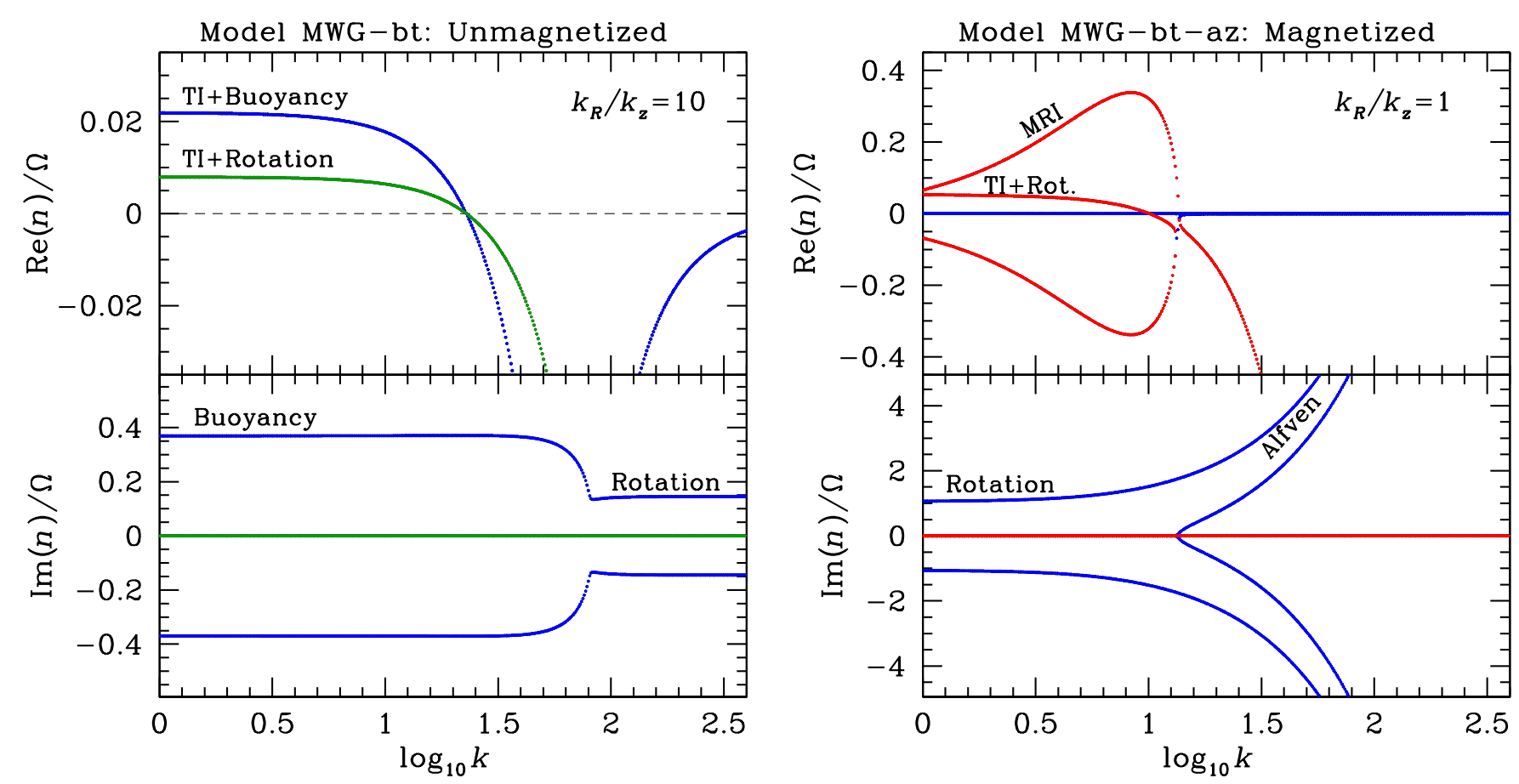

Figure 5. Same as Fig. 4, but, in the left-hand panel, for model MWG-bt with no magnetic field and $k_{R} / k_{z}=10$ (isotropic conduction with $f=0.01$, dispersion relation 16), and, in the right-hand panel, for the magnetized model MWG-bt-az and $k_{R} / k_{z}=1$ (anisotropic conduction, dispersion relation 6). The branches are identified as in Fig. 4, with the addition of the magnetorotational instability (MRI) mode. 


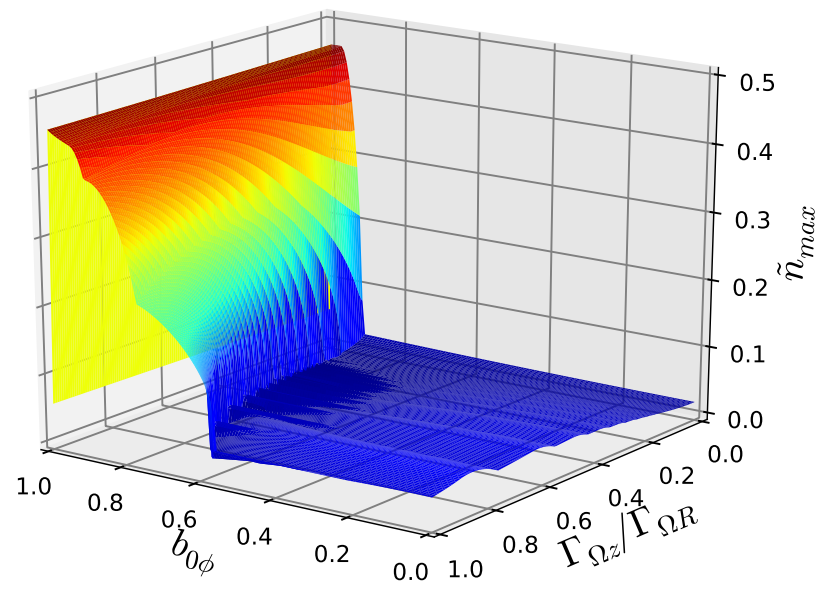

Figure 6. Maximum growth rate (in units of $\Omega$ ) of monotonically unstable modes as a function of the azimuthal component of the magnetic field $b_{0 \phi}$ and of $\Gamma_{\Omega z} / \Gamma_{\Omega R}$, which is a measure of the vertical-to-radial velocity gradient ratio, for a set of magnetized Milky-Way galaxy models. The models considered here have the same values of the parameters $\Gamma_{\Omega R}, \Gamma_{p R}, \Gamma_{p z}, \Gamma_{T R}, \tilde{c}_{0}, \tilde{\omega}_{\text {th }}, \tilde{\omega}_{\mathrm{c}, 0}$, and $\beta$ as models MWG (see Table 1), but different values of $\Gamma_{\Omega z}$ and $b_{0 \phi}$ (and, as a consequence, different values of the dependent parameters $b_{0 R}, b_{0 z}$ and $\left.\Gamma_{T z}\right)$. We explored wave-vectors with $10 \leq k R \leq 1000$ and $-25 \leq k_{R} / k_{z} \leq 25$.

unstable branches, we note that in model MWG-bt (in which temperature decreases outwards; $\nabla p_{0} \cdot \nabla T_{0}>0$ ) the monotonically unstable mode is due to a combination of MTI and TI. However, we verified that the growth rate is dominated by the TI: the MTI is weaker, because the temperature is relatively low and the magnetic field lines are non-isothermal (see Quataert 2008), and the instability is essentially a TI. In model CCC-bt (in which temperature increases outwards; $\nabla p_{0} \cdot \nabla T_{0}<0$ ) the monotonically unstable mode is due to a combination of HBI and TI, where the HBI is dominant (because of the high temperature). In both models there are two over-stable branches (due to a combination of TI with either rotation or buoyancy), but these modes occur at sufficiently short wave-lengths $(k R \gtrsim 10)$ only for model MWG. We note that in the over-stable modes the buoyancy frequency is much higher than the TI frequency, so over-stable modes are not expected to lead to local condensation (Malagoli et al. 1987). However, the properties of the monotonically growing modes suggest that local condensation via TI should be more likely in galactic atmospheres than in cool cores of galaxy clusters. A comparison with the roots obtained for model MWG-bt with no magnetic field (in which only over-stable TI modes are present and the oscillating branches are buoyancy and rotation modes; Fig. 5, left-hand panel) suggests that the presence of ordered magnetic field tends to promote local condensation through the combination of the TI with the either the MTI or the HBI.

What is the role of rotation? In Section 3.4.1 we anticipated that in some cases the instability is dominated the MRI. An example is given in the right-hand panel of Fig. 5, showing the roots for model MWG-bt-az fixing $x=1$ : it is apparent that the strongest monotonic instability (maximum growth rate $n / \Omega \approx 0.35$ ) is not thermal, but MRI. We note that all the plasma models analyzed in this work are formally MRI unstable because the angular velocity decreases outwards while the entropy increases outwards (see Balbus \& Hawley 1991). However, the MRI occurs only for $k$ smaller than a critical value $k_{\mathrm{MRI}}$ (Balbus \& Hawley 1991), which in the considered cases is typically lower than our fiducial limit for short wavelength $k=10 R^{-1}$. In our models only when $b_{0 \phi}$ is close to unity the MRI occurs at relevant wavelengths, basically because $k_{\mathrm{MRI}}$ increases when the magnetic field components coupled with the axisymmetric perturbation $\left(B_{0 z}\right.$ and $\left.B_{0 R}\right)$ decrease. We note that in this case the branch due to the combination of TI and rotation is monotonically growing, but with substantially smaller growth rate than the MRI. We expect that these MRI dominated modes do not lead to local condensation. Even when the MRI is not dominant, the effect of rotation on the instabilities studied in this paper is very important. In the presence of rotation, at least in the simple configurations here considered in which all the components of the background magnetic field are time-independent, the magnetic field lines lie on surfaces of constant angular velocity, which, even in the absence of cooling, favors the onset of the HBI or of the MTI. In the presence of cooling the combination of the TI with either the HBI or the MTI gives rise to monotonically unstable modes, which are expected to lead to local condensation, provided the plasma temperature is not too high (otherwise the HBI and the MTI dominate).

The numerical values of the growth rates give additional information on the astrophysical implications of the studied instabilities. In our reference range of wave-vector components $(10 \leq k R \leq 1000,-25<x<25)$ the maximum growth rate $\tilde{n}_{\max } \equiv \max [\operatorname{Re}(n)] / \Omega$ of monotonically growing and over-stable modes $[\operatorname{Re}(n)>0]$ does not exceed $\tilde{n}_{\max } \approx 0.04$ for models MWG with $b_{0 \phi}=0$. In the models with azimuthal field $\left(b_{0 \phi}=0.95\right)$ we get substantially higher growth rates, with $\tilde{n}_{\max } \approx 0.44$, due to the onset of the MRI. For models CCC we find $\tilde{n}_{\max } \approx 0.14$ for monotonic instability (which is dominated by the HBI) and $\tilde{n}_{\max } \approx 0.03$ for over-stability (dominated by the TI). Therefore, in the models here considered, even for the fastest-growing TI modes the growth-timescale (which is related to the cooling time) is typically more than one order of magnitude longer than $\Omega^{-1}$ (which is related to the dynamical time). Over shorter timescales we expect the HBI to occur in cool cores and, in specific conditions, the MRI in galactic coronae (and 
possibly also in clusters). An overview of the instability growth rate in a magnetized, rotating Milky-Way like galaxy corona is given in Fig. 6, where we plot the maximum growth-rate of monotonically unstable modes $\tilde{n}_{\text {max }}$ as a function of $b_{0 \phi}$ and $\Gamma_{\Omega z} / \Gamma_{\Omega R}$ for a set of models, which is an extension of the set MWG. We note that unstable and over-stable modes are present in all models of this extended set $\left(0 \leq b_{0 \phi} \leq 1\right.$ and $\left.0 \leq \Gamma_{\Omega z} / \Gamma_{\Omega R} \leq 1\right)$. When $b_{0 \phi} \rightarrow 1$ the growth-rates are maximized (up to $\tilde{n}_{\max } \approx 0.45$ ), because the MRI is dominant, but when $b_{\phi}=1$ exactly the growth rate drops by one order of magnitude, because there is no axisymmetric MRI if $b_{0 R}=b_{0 z}=0$. Figure 6 also shows that varying $\Gamma_{\Omega z} / \Gamma_{\Omega R}$ has generally a small effect on the maximum growth rate, though the trend is that $\tilde{n}_{\text {max }}$ is larger for larger vertical velocity gradients, so baroclinic models tend to be more unstable than barotropic models (as well known, vertical velocity gradients are destabilizing even in the absence of magnetic fields; see, e.g., Nelson et al. 2013 and references therein).

For both MWG and CCC models, we have explored the effect of varying the thermal-to-magnetic pressure ratio $\beta$ : the general trend is that the areas of monotonic instability and over-stability increase for increasing $\beta$, at the expense of the areas of stability. However, the effect on the growth rates is small for variations of $\beta$ up to a factor of a few. Remarkably, if $\beta$ is increased by one order of magnitude or more the MRI tends to be dominant in all models $\left(k_{\mathrm{MRI}} \gg R^{-1}\right)$.

\section{NON-AXISYMMETRIC PERTURBATIONS}

Here we extend the above linear stability analysis to the case of non-axisymmetric perturbations. Though formally the existence of unstable axisymmetric modes is sufficient to infer that the system is locally unstable, physically it is important to establish whether the assumption of axisymmetry is a key factor for the onset of the instability. For instance, in the unmagnetized case, differentially rotating systems that are thermally unstable against axisymmetric perturbations tend to be stable against non-axisymmetric disturbances (N10).

\subsection{Derivation of the system of ordinary differential equations}

The evolution of non-axisymmetric perturbations in a differentially rotating plasma is more complicated than the axisymmetric case, because of the effect of the shear (see, e.g., Cowling 1951; Lin \& Shu 1964; Goldreich \& Lynden-Bell 1965; Bertin et al. 1989; Balbus \& Hawley 1992). As in N10, we adopt here shearing coordinates $\phi^{\prime}=\phi-\Omega(R, z) t$, $R^{\prime}=R, z^{\prime}=z$ and $t^{\prime}=t$ (Goldreich \& Lynden-Bell 1965; Balbus \& Hawley 1992) to reduce the problem to a system of ordinary differential equations (ODEs). In the primed coordinates the non-axisymmetric perturbations take the form of plane waves, so we can write the perturbed quantities as $F_{0}+F\left(t^{\prime}\right) \exp \left(\mathrm{i} k_{R}^{\prime} R^{\prime}+\mathrm{i} k_{z}^{\prime} z^{\prime}+\mathrm{i} m \phi^{\prime}\right)$, with $k_{R}^{\prime}, k_{z}^{\prime}$ and $m$ constant. Linearizing equations (1-4) in the Boussinesq approximation (so $T / T_{0} \simeq-\rho / \rho_{0}$ ) we get

$$
\begin{aligned}
& k_{R} v_{R}+k_{z} v_{z}+k_{\phi} v_{\phi}=0, \\
& \rho_{0} \frac{\mathrm{d} v_{R}}{\mathrm{~d} t^{\prime}}-2 \Omega \rho_{0} v_{\phi}+\mathrm{i} k_{R} p-A_{p R} c_{0}^{2} \rho-\frac{\mathrm{i}}{4 \pi}\left(\mathbf{k} \cdot \mathbf{B}_{0}\right) B_{R}+\frac{\mathrm{i} k_{R}}{4 \pi}\left(\mathbf{B}_{0} \cdot \mathbf{B}\right)=0, \\
& \rho_{0} \frac{\mathrm{d} v_{\phi}}{\mathrm{d} t^{\prime}}+\left(2 \Omega+R^{\prime} \frac{\partial \Omega}{\partial R}\right) \rho_{0} v_{R}+R^{\prime} \frac{\partial \Omega}{\partial z} \rho_{0} v_{z}+\mathrm{i} k_{\phi} p-\frac{\mathrm{i}}{4 \pi}\left(\mathbf{k} \cdot \mathbf{B}_{0}\right) B_{\phi}+\frac{\mathrm{i} k_{\phi}}{4 \pi}\left(\mathbf{B}_{0} \cdot \mathbf{B}\right)=0, \\
& \rho_{0} \frac{\mathrm{d} v_{z}}{\mathrm{~d} t^{\prime}}+\mathrm{i} k_{z} p-A_{p z} c_{0}^{2} \rho-\frac{\mathrm{i}}{4 \pi}\left(\mathbf{k} \cdot \mathbf{B}_{0}\right) B_{z}+\frac{\mathrm{i} k_{z}}{4 \pi}\left(\mathbf{B}_{0} \cdot \mathbf{B}\right)=0, \\
& \frac{\mathrm{d} B_{R}}{\mathrm{~d} t^{\prime}}-\mathrm{i}\left(\mathbf{k} \cdot \mathbf{B}_{0}\right) v_{R}=0, \\
& \frac{\mathrm{d} B_{\phi}}{\mathrm{d} t^{\prime}}-\mathrm{i}\left(\mathbf{k} \cdot \mathbf{B}_{0}\right) v_{\phi}-R^{\prime} \nabla \Omega \cdot \mathbf{B}=0, \\
& \frac{\mathrm{d} B_{z}}{\mathrm{~d} t^{\prime}}-\mathrm{i}\left(\mathbf{k} \cdot \mathbf{B}_{0}\right) v_{z}=0, \\
& -\frac{\gamma}{\rho_{0}} \frac{\mathrm{d} \rho}{\mathrm{d} t^{\prime}}+\left(A_{p R}-\gamma A_{\rho R}\right) v_{R}+\left(A_{p z}-\gamma A_{\rho z}\right) v_{z} \\
& \quad-\frac{\gamma-1}{p_{0}}\left[\frac{\chi\left(T_{0}\right) T_{0}\left(\mathbf{k} \cdot \mathbf{b}_{0}\right)^{2}}{\rho_{0}}-\left[\mathcal{L}\left(\rho_{0}, T_{0}\right)+\rho_{0} \mathcal{L}_{\rho}\left(\rho_{0}, T_{0}\right)-T_{0} \mathcal{L}_{T}\left(\rho_{0}, T_{0}\right)\right]\right] \\
& \quad-\mathrm{i} \frac{(\gamma-1) \chi\left(T_{0}\right) T_{0}}{p_{0}}\left[\left(\mathbf{k} \cdot \mathbf{b}_{0}\right) \nabla \ln T_{0}-2\left(\mathbf{b}_{0} \cdot \mathbf{k}\right)\left(\nabla \ln T_{0} \cdot \mathbf{b}_{0}\right) \mathbf{b}_{0}\right] \cdot \mathbf{b}=0,
\end{aligned}
$$

where we have introduced the quantities

$$
A_{p R} \equiv \frac{1}{p_{0}} \frac{\partial p_{0}}{\partial R}, \quad A_{p z} \equiv \frac{1}{p_{0}} \frac{\partial p_{0}}{\partial z}, \quad A_{\rho R} \equiv \frac{1}{\rho_{0}} \frac{\partial \rho_{0}}{\partial R}, \quad A_{\rho z} \equiv \frac{1}{\rho_{0}} \frac{\partial \rho_{0}}{\partial z},
$$

and $\mathbf{k}=\mathbf{k}\left(t^{\prime}\right) \equiv\left(k_{R}, k_{\phi}, k_{z}\right)$ is the time-dependent wave-vector, with

$$
k_{R}=k_{R}\left(t^{\prime}\right)=k_{R}^{\prime}-m t^{\prime} \frac{\partial \Omega}{\partial R},
$$




$$
k_{z}=k_{z}\left(t^{\prime}\right)=k_{z}^{\prime}-m t^{\prime} \frac{\partial \Omega}{\partial z}
$$

and $k_{\phi} \equiv m / R^{\prime}$ (independent of time). In equation (38) we have used the divergence-free condition $\nabla \cdot \mathbf{B}=0$ to eliminate the term proportional to $\mathbf{k} \cdot \mathbf{b}$. The system of ODEs (31-38) can be simplified by eliminating the variables $v_{\phi}$, $p$ and $B_{\phi}$. In particular, in the momentum equations, $v_{\phi}$ can be eliminated by using the mass conservation equation $v_{\phi}=-\left(k_{R} v_{R}+k_{z} v_{z}\right) / k_{\phi}$ and its time derivative ${ }^{3}$

$$
\frac{\mathrm{d} v_{\phi}}{\mathrm{d} t^{\prime}}=-\frac{k_{R}}{k_{\phi}} \frac{\mathrm{d} v_{R}}{\mathrm{~d} t^{\prime}}-\frac{k_{z}}{k_{\phi}} \frac{\mathrm{d} v_{z}}{\mathrm{~d} t^{\prime}}+R \frac{\partial \Omega}{\partial R} v_{R}+R \frac{\partial \Omega}{\partial z} v_{z}
$$

The pressure perturbation $p$ can be eliminated by combining the momentum equations as follows: we subtract the $z$ equation (multiplied by $k_{R}$ ) from the $R$ equation (multiplied by $k_{z}$ ), and we subtract the $z$ equation (multiplied by $k_{\phi}$ ) from the $\phi$ equation (multiplied by $k_{z}$ ), so

$$
\mathrm{i} k_{z} p=-\rho_{0} \frac{\mathrm{d} v_{z}}{\mathrm{~d} t^{\prime}}+A_{p z} c_{0}^{2} \rho+\frac{\mathrm{i}}{4 \pi}\left(\mathbf{k} \cdot \mathbf{B}_{0}\right) B_{z}-\frac{\mathrm{i} k_{z}}{4 \pi}\left(\mathbf{B}_{0} \cdot \mathbf{B}\right) .
$$

The divergence-free condition $\nabla \cdot \mathbf{B}=0$ (i.e. $\mathbf{k} \cdot \mathbf{B}=0$ ) can be used to eliminate $B_{\phi}$, which can be written as

$$
B_{\phi}=-\frac{k_{R}}{k_{\phi}} B_{R}-\frac{k_{z}}{k_{\phi}} B_{z}
$$

After rearrangement and simplification, we end up with the following system of five ODEs (in the variables $v_{R}, v_{z}, \rho$, $B_{R}$ and $\left.B_{z}\right)$ :

$$
\begin{aligned}
& \frac{\mathrm{d} v_{R}}{\mathrm{~d} t^{\prime}}=\frac{2 k_{R} k_{\phi} \Omega}{k^{2}}\left(\Gamma_{\Omega R}-\frac{k_{z}^{2}}{k_{\phi}^{2}}\right) v_{R}+\frac{2 k_{R} k_{\phi} \Omega}{k^{2}}\left(\Gamma_{\Omega z}-\frac{k_{z}}{k_{R}} \frac{k_{z}^{2}+k_{\phi}^{2}}{k_{\phi}^{2}}\right) v_{z}+\frac{c_{0}^{2} A_{p R}}{\rho_{0}}\left(\frac{k_{z}^{2}+k_{\phi}^{2}}{k^{2}}-\frac{A_{p z}}{A_{p R}} \frac{k_{R} k_{z}}{k^{2}}\right) \rho \\
&+\frac{\mathrm{i}}{4 \pi \rho_{0}}\left(\mathbf{k} \cdot \mathbf{B}_{0}\right) B_{R}, \\
& \frac{\mathrm{d} v_{z}}{\mathrm{~d} t^{\prime}}=\frac{2 k_{z} k_{\phi} \Omega}{k^{2}}\left(\Gamma_{\Omega R}+\frac{k_{R}^{2}+k_{\phi}^{2}}{k_{\phi}^{2}}\right) v_{R}+\frac{2 k_{z} k_{\phi} \Omega}{k^{2}}\left(\Gamma_{\Omega z}+\frac{k_{z} k_{R}}{k_{\phi}^{2}}\right) v_{z}+\frac{c_{0}^{2} A_{p z}}{\rho_{0}}\left(\frac{k_{R}^{2}+k_{\phi}^{2}}{k^{2}}-\frac{A_{p R}}{A_{p z}} \frac{k_{R} k_{z}}{k^{2}}\right) \rho \\
&+\frac{\mathrm{i}}{4 \pi \rho_{0}}\left(\mathbf{k} \cdot \mathbf{B}_{0}\right) B_{z}, \\
& \frac{\mathrm{d} B_{R}}{\mathrm{~d} t^{\prime}}-\mathrm{i}\left(\mathbf{k} \cdot \mathbf{B}_{0}\right) v_{R}=0, \\
& \frac{\mathrm{d} B_{z}}{\mathrm{~d} t^{\prime}}-\mathrm{i}\left(\mathbf{k} \cdot \mathbf{B}_{0}\right) v_{z}=0, \\
&-\frac{\gamma}{\rho_{0}} \frac{\mathrm{d} \rho}{\mathrm{d} t^{\prime}}+\left(A_{p R}-\gamma A_{\rho R}\right) v_{R}+\left(A_{p z}-\gamma A_{\rho z}\right) v_{z} \\
& \quad-\frac{\gamma-1}{p_{0}}\left[\frac{\chi\left(T_{0}\right) T_{0}\left(\mathbf{k} \cdot \mathbf{b}_{0}\right)^{2}}{\rho_{0}}-\left[\mathcal{L}\left(\rho_{0}, T_{0}\right)+\rho_{0} \mathcal{L}_{\rho}\left(\rho_{0}, T_{0}\right)-T_{0} \mathcal{L}_{T}\left(\rho_{0}, T_{0}\right)\right]\right] \\
& \quad-\mathrm{i} \frac{(\gamma-1) \chi\left(T_{0}\right) T_{0}\left(\mathbf{k} \cdot \mathbf{b}_{0}\right)}{p_{0}}\left[\nabla \ln T_{0}-2\left(\nabla \ln T_{0} \cdot \mathbf{b}_{0}\right) \mathbf{b}_{0}\right] \cdot \mathbf{b}=0 .
\end{aligned}
$$

Dividing equations (45) and (46) by $\Omega^{2} R$, equations (47) and (48) by $B_{0} \Omega$, and equation (49) by $\gamma \Omega$, the above system can be rewritten in dimensionless form as

$$
\begin{aligned}
& \frac{\mathrm{d} \tilde{v}_{R}}{\mathrm{~d} \tau}=X_{R R} \tilde{v}_{R}+X_{R z} \tilde{v}_{z}+X_{R \rho} \tilde{\rho}+\frac{2 \mathrm{i} K \tilde{c}_{0}^{2}}{\beta} b_{R} \\
& \frac{\mathrm{d} \tilde{v}_{z}}{\mathrm{~d} \tau}=X_{z R} \tilde{v}_{R}+X_{z z} \tilde{v}_{z}+X_{z \rho} \tilde{\rho}+\frac{2 \mathrm{i} K \tilde{c}_{0}^{2}}{\beta} b_{z} \\
& \frac{\mathrm{d} b_{R}}{\mathrm{~d} \tau}=\mathrm{i} K \tilde{v}_{R}, \\
& \frac{\mathrm{d} b_{z}}{\mathrm{~d} \tau}=\mathrm{i} K \tilde{v}_{z}, \\
& \frac{\mathrm{d} \tilde{\rho}}{\mathrm{d} \tau}=X_{\rho R} \tilde{v}_{R}+X_{\rho z} \tilde{v}_{z}+X_{\rho \rho} \tilde{\rho}-\frac{2 i K \tilde{c}_{0}^{2}}{\beta} Y_{\rho R} b_{R}-\frac{2 i K \tilde{c}_{0}^{2}}{\beta} Y_{\rho z} b_{z},
\end{aligned}
$$

3 The correct expression for $\mathrm{d} v_{\phi} / \mathrm{d} t^{\prime}$ is the one reported here and not that given in equation 60 of N10, where the last two terms are missing because of a typographical error. However, the correct expression for $\mathrm{d} v_{\phi} / \mathrm{d} t^{\prime}$ is used in all the calculations of N10. 
where, for simplicity we have assumed $b_{0 \phi}=0$ and so eliminated the term proportional to $b_{0 \phi} b_{\phi}$ in the energy equation. In the above equations $\tilde{v}_{R} \equiv v_{R} / \Omega R, \tilde{v}_{z} \equiv v_{z} / \Omega R, \tilde{\rho} \equiv \rho / \rho_{0}, \tau \equiv t^{\prime} \Omega, K \equiv\left(\tilde{\mathbf{k}} \cdot \mathbf{b}_{0}\right) m, \tilde{k}_{R} \equiv k_{R} / k_{\phi}, \tilde{k}_{z} \equiv k_{z} / k_{\phi}$, $\tilde{k} \equiv k / k_{\phi}, m=k_{\phi} R^{\prime}$, and the quantities indicated with $X$ and $Y$ (defined in Appendix A) are dimensionless functions of $\tilde{\mathbf{k}}(\tau)$ and of the fluid parameters. In dimensionless variables we have

$$
\tilde{k}_{R}(\tau)=\tilde{k}_{R}^{\prime}-\tau \Gamma_{\Omega R} \text { and } \tilde{k}_{z}(\tau)=\tilde{k}_{z}^{\prime}-\tau \Gamma_{\Omega z}
$$

so $\mathrm{d} \tilde{k}_{R} / \mathrm{d} \tau=-\Gamma_{\Omega R}$ and $\mathrm{d} \tilde{k}_{z} / \mathrm{d} \tau=-\Gamma_{\Omega z}$. It is useful to note that from the assumed condition of isorotational background field $\left(\nabla \Omega \cdot \mathbf{B}_{0}=0\right)$ it follows that $\mathrm{d}\left(\mathbf{k} \cdot \mathbf{b}_{0}\right) / \mathrm{d} \tau=0$, so $K$ is constant. Differentiating equations (50) and (51) with respect to $\tau$ and using equations (52) and (53) in the form i $K \mathrm{~d} b_{R} / \mathrm{d} \tau=-K^{2} v_{R}$ and $\mathrm{i} K \mathrm{~d} b_{z} / \mathrm{d} \tau=-K^{2} v_{z}$, we get the following system of ODEs in the variables $\tilde{v}_{R}, \tilde{v}_{z}$ and $\tilde{\rho}$ :

$$
\begin{aligned}
& \frac{\mathrm{d}^{2} \tilde{v}_{R}}{\mathrm{~d} \tau^{2}}=\left(X_{R R}-X_{R \rho} Y_{\rho R}\right) \frac{\mathrm{d} \tilde{v}_{R}}{\mathrm{~d} \tau}+\left(X_{R z}-X_{R \rho} Y_{\rho z}\right) \frac{\mathrm{d} \tilde{v}_{z}}{\mathrm{~d} \tau}+\left[X_{R \rho}\left(X_{\rho R}+X_{R R} Y_{\rho R}+X_{z R} Y_{\rho z}\right)+\dot{X}_{R R}-\frac{2 K^{2} \tilde{c}_{0}^{2}}{\beta}\right] \tilde{v}_{R} \\
&+ {\left[X_{R \rho}\left(X_{\rho z}+X_{R z} Y_{\rho R}+X_{z z} Y_{\rho z}\right)+\dot{X}_{R z}\right] \tilde{v}_{z}+\left[X_{R \rho}\left(X_{\rho \rho}+X_{R \rho} Y_{\rho R}+X_{z \rho} Y_{\rho z}\right)+\dot{X}_{R \rho}\right] \tilde{\rho}, } \\
& \frac{\mathrm{d}^{2} \tilde{v}_{z}}{\mathrm{~d} \tau^{2}}=\left(X_{z R}-X_{z \rho} Y_{\rho R}\right) \frac{\mathrm{d} \tilde{v}_{R}}{\mathrm{~d} \tau}+\left(X_{z z}-X_{z \rho} Y_{\rho z}\right) \frac{\mathrm{d} \tilde{v}_{z}}{\mathrm{~d} \tau}+\left[X_{z \rho}\left(X_{\rho R}+X_{R R} Y_{\rho R}+X_{z R} Y_{\rho z}\right)+\dot{X}_{z R}\right] \tilde{v}_{R} \\
&+\left[X_{z \rho}\left(X_{\rho z}+X_{R z} Y_{\rho R}+X_{z z} Y_{\rho z}\right)+\dot{X}_{z z}-\frac{2 K^{2} \tilde{c}_{0}^{2}}{\beta}\right] \tilde{v}_{z}+\left[X_{z \rho}\left(X_{\rho \rho}+X_{R \rho} Y_{\rho R}+X_{z \rho} Y_{\rho z}\right)+\dot{X}_{z \rho}\right] \tilde{\rho}, \\
& \frac{\mathrm{d} \tilde{\rho}}{\mathrm{d} \tau}=-Y_{\rho R} \frac{\mathrm{d} \tilde{v}_{R}}{\mathrm{~d} \tau}-Y_{\rho z} \frac{\mathrm{d} \tilde{v}_{z}}{\mathrm{~d} \tau}+\left(X_{\rho R}+X_{R R} Y_{\rho R}+X_{z R} Y_{\rho z}\right) \tilde{v}_{R}+\left(X_{\rho z}+X_{R z} Y_{\rho R}+X_{z z} Y_{\rho z}\right) \tilde{v}_{z} \\
&+\left(X_{\rho \rho}+X_{R \rho} Y_{\rho R}+X_{z \rho} Y_{\rho z}\right) \tilde{\rho},
\end{aligned}
$$

where the quantities indicated with $\dot{X}$ (reported in Appendix A) are the derivatives with respect to $\tau$ of the corresponding dimensionless functions indicated with $X$ that we introduced in equations (50-51). The final system of ODEs (56-58) in the variables $\tilde{v}_{R}, \tilde{v}_{z}$ and $\tilde{\rho}$ fully describes the evolution of non-axisymmetric perturbations. The system must be completed by specifying at $\tau=0$ the values of $\tilde{v}_{R}, \tilde{v}_{z}, \tilde{\rho}, \mathrm{d} \tilde{v}_{R} / \mathrm{d} \tau$ and $\mathrm{d} \tilde{v}_{z} / \mathrm{d} \tau$, which must be such that equations (50-51) are satisfied at $\tau=0$. The coefficients of the system of equations (56-58) depend on the following

parameters: $\tilde{k}_{R}^{\prime}, \tilde{k}_{z}^{\prime}, m$ (characterizing the perturbation), $\Gamma_{\Omega R}, \Gamma_{\Omega z}, \Gamma_{p R}, \Gamma_{p z}, \Gamma_{T R}, \tilde{c}_{0}, \tilde{\omega}_{\mathrm{th}}, \tilde{\omega}_{\mathrm{c}, 0}, \beta$ (defining the properties of the background fluid; we recall that here we are assuming $\left.b_{0 \phi}=0\right)$. The other parameters appearing in the coefficients (see Appendix A) are not independent (see Section 3.2). In particular, we recall that $\Gamma_{\rho R}=\Gamma_{p R}-\Gamma_{T R}$ and $\Gamma_{\rho z}=\Gamma_{p z}-\Gamma_{T z}$.

\subsection{Results for non-axisymmetric perturbations}

Given a plasma model, the evolution of a given non-axisymmetric mode can be calculated by numerically integrating the system of ODEs (56-58). In particular, we have solved the system with a fourth-order Runge-Kutta method for the models MWG-bt, MWG-bc, CCC-bt and CCC-bc, exploring a wide range of values of wave numbers $\left(\tilde{k}_{R}^{\prime}\right.$, $\left.\tilde{k}_{z}^{\prime}, m\right)$ and initial conditions $\left[\tilde{v}_{R}(0), \tilde{v}_{z}(0), \tilde{\rho}(0), \mathrm{d} \tilde{v}_{R} / \mathrm{d} \tau(0), \mathrm{d} \tilde{v}_{z} / \mathrm{d} \tau(0)\right]$. Overall, the results of these numerical calculations show that, in the presence of ordered magnetic field (and therefore anisotropic thermal conduction), the evolution of non-axisymmetric perturbations with initial wave-vector components $k_{R}, k_{z}$ and $k=\left(k_{R}^{2}+k_{z}^{2}+k_{\phi}^{2}\right)^{1 / 2}$ is qualitatively similar to that of axisymmetric perturbations with the same values of $k_{R} / k_{z}$ and $k$. This indicates that the instabilities found in NP13 and analyzed in Section 3 are not due to the axisymmetry of the disturbance and confirms that the considered rotating plasmas are locally unstable to general perturbations. Remarkably, this is different from the unmagnetized case, in which non-axisymmetric perturbations are typically stable even if the corresponding axisymmetric perturbations are unstable (N10).

The above finding is illustrated in Fig. 7, showing the evolution of a representative non-axisymmetric perturbation in the magnetized (anisotropic conduction; left-hand panel) and unmagnetized (isotropic conduction; right-hand panel) model MWG-bc (for the unmagnetized system the numerical solution is obtained as described in N10; equations 64-66 in that paper). In both cases the initial wave vector components $\tilde{k}_{R}=0.5, \tilde{k}_{z}=0.1, m=10$ (i.e. $x=5$, $k \simeq 11.2$ ) correspond to unstable regions for axisymmetric perturbations (see Section 3.4.1) and the initial conditions are $\tilde{v}_{R}(0)=0.01, \tilde{v}_{z}(0)=-0.01$ and $\tilde{\rho}(0)=0.01$. In the magnetized model the two additional initial conditions $\mathrm{d} \tilde{v}_{R} / \mathrm{d} \tau(0)$ and $\mathrm{d} \tilde{v}_{z} / \mathrm{d} \tau(0)$ are such that equations (50-51) are satisfied with $\mathbf{b}=0$ at $\tau=0$. From Fig. 7 it is apparent that the non-axisymmetric perturbation is unstable (it enters the non-liner regime at $\tau=t \Omega \simeq 45$ ) when the medium is magnetized, while it is manifestly stable when the system is unmagnetized, due to isotropic conduction (even if suppressed by a factor $f=0.01$ ).

Physically, the different behavior of non-axisymmetric disturbances in magnetized and unmagnetized fluids can be understood by considering the simple case of the evolution of a blob-like (non-axisymmetric) thermal perturbation in the presence of differential rotation $\Omega=\Omega(R)$ (see Fig. 8). The blob is a small overdensity close to pressure equilibrium with the hotter surrounding medium. The effect of the shear is to stretch the perturbation along the 

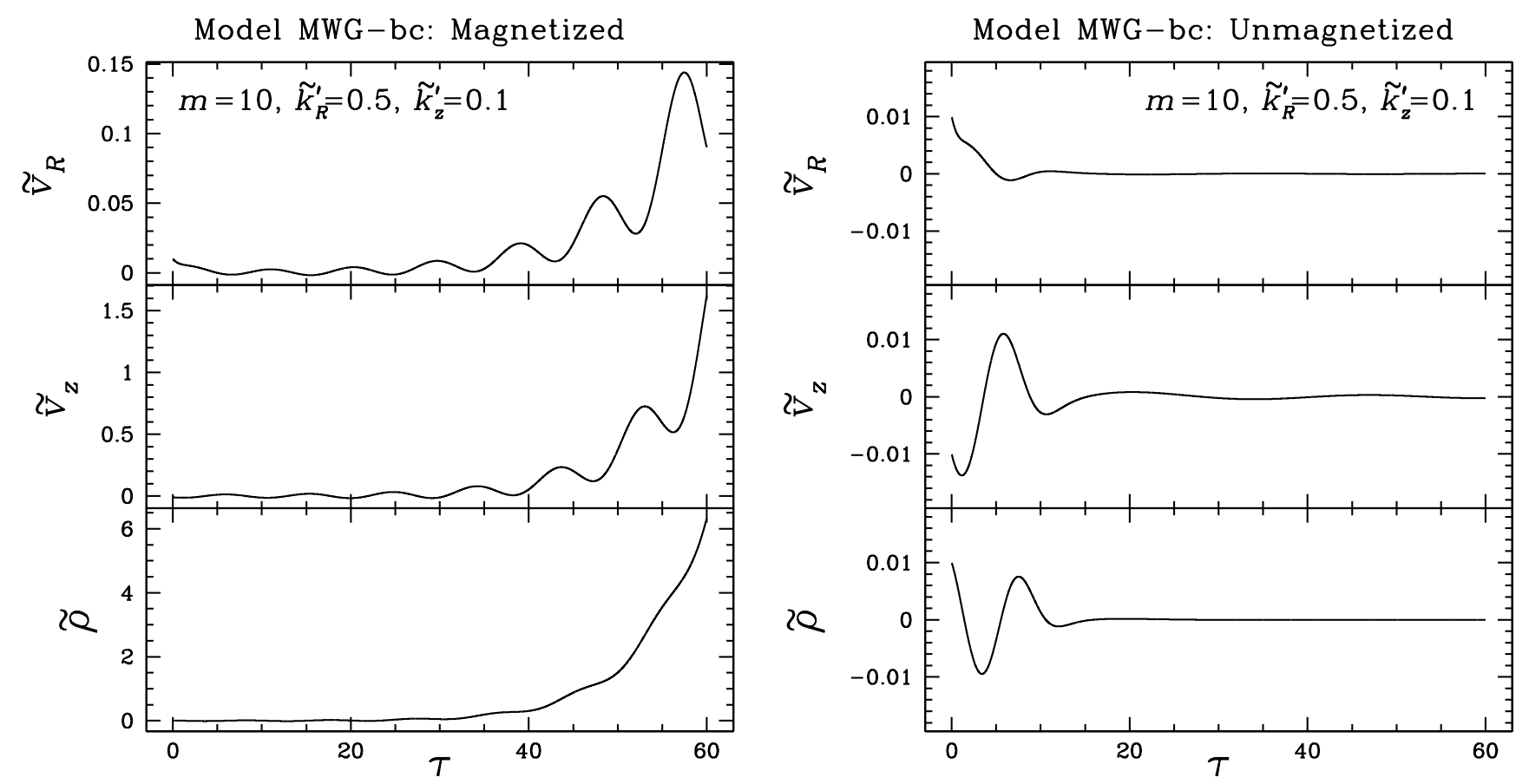

Figure 7. Evolution of a non-axisymmetric perturbation for the baroclinic Milky-Way like galaxy model MWG-bc in the presence (left-hand panel) and in the absence (right-hand panel) of magnetic field. Here $\tilde{\rho}, \tilde{v}_{z}$ and $\tilde{v}_{R}$ are the normalized density and velocity perturbations (see Section 4.1); $\tau$ is the time in units of $\Omega^{-1}$. Note the different scales in the vertical axes in the two panels. In the unmagnetized case we have assumed thermal conduction suppression factor $f=0.01$.

\section{Magnetized, anisotropic conduction}

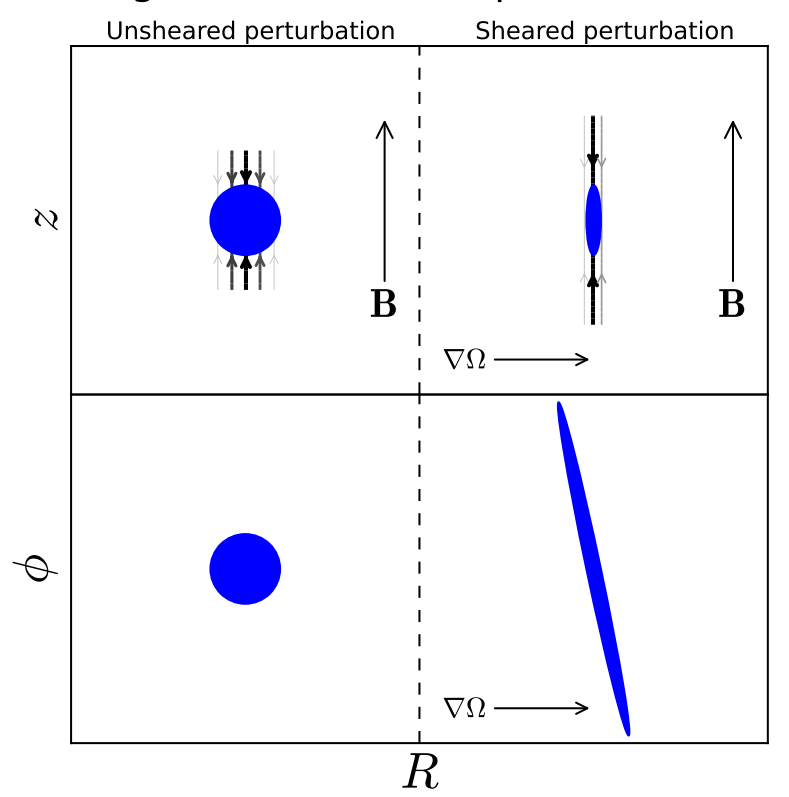

\section{Unmagnetized, isotropic conduction}

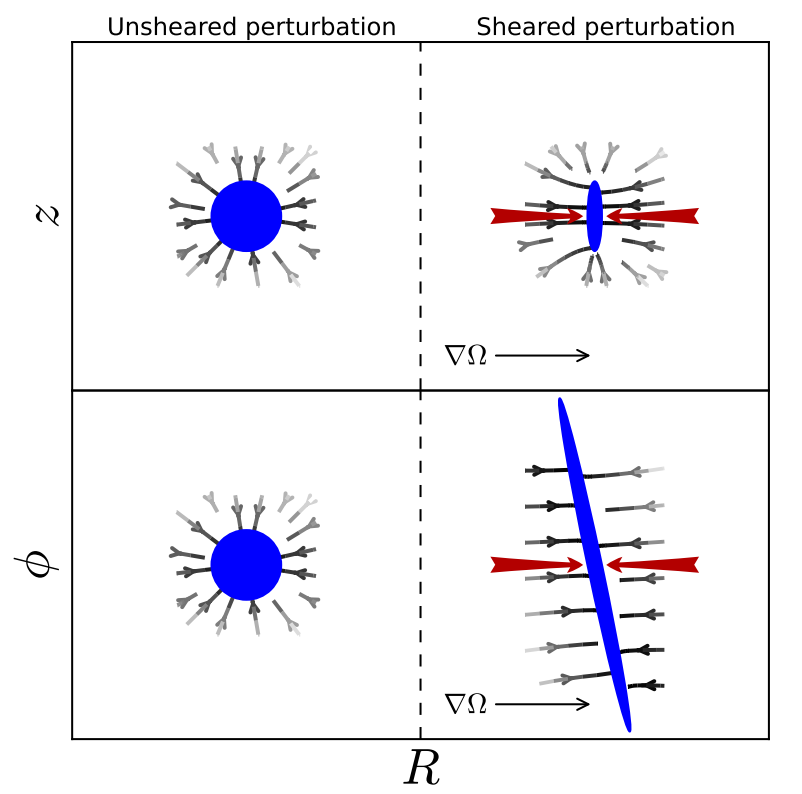

Figure 8. Left-hand panel: a schematic representation of the evolution of a blob-like (non-axisymmetric) thermal disturbance (colder than the background) in the presence of differential rotation $\Omega=\Omega(R)$ and isorotational magnetic field with no azimuthal component. The four quadrants show sections of the initial (unsheared; left) and evolved (sheared; right) perturbation in the planes $R-\phi$ and $R$ - $z$. The conductive heat flux $\mathbf{Q}$ (small gray arrows) is anisotropic and it is null across the magnetic field lines (i.e. along $R$ ), so the sheared perturbation is not damped. Here we neglect the (small) perturbation in the magnetic field direction. Right-hand panel: same as the left-hand panel, but for an unmagnetized medium. In this case $\mathbf{Q}$ is isotropic, so it can effectively damp the sheared thermal perturbation, which is narrow along $R$ (as indicated by the big red arrows). 
azimuthal direction making it narrow along $R$ (mathematically, $k_{R}$ increases with time). In the magnetized case we have $B_{0 R}=0$ (due to the isorotation condition $\mathbf{B}_{0} \cdot \nabla \Omega=0$ ), so, neglecting the small perturbation in the magnetic field direction, heat conduction does not occur along $R$ and the perturbation is not damped (left-hand panel in Fig. 8, where for simplicity we assume that the azimuthal magnetic field component is null). In the absence of magnetic field thermal conduction is isotropic, so it is effective along the radial direction and can easily damp the thermal disturbance which gets thinner because of the shear (right-hand panel in Fig. 8).

\section{SUMMARY AND CONCLUSIONS}

In this paper we have studied the nature of local instabilities in rotating, stratified, weakly magnetized, optically thin astrophysical plasmas in the presence of radiative cooling and anisotropic thermal conduction. A summary of the main results of the present work is the following.

1. We have provided the equations that allow to determine the linear evolution of axisymmetric and nonaxisymmetric perturbations at any position of a differentially rotating plasma in the presence of a weak ordered magnetic field. Given a model for the background plasma, the evolution of axisymmetric perturbations can be computed by solving numerically the dispersion relation (6). The evolution of non-axisymmetric perturbations can be determined by integrating numerically the system of ODEs (56-58).

2. We have studied the stability properties of rotating models representative of cool cores of galaxy clusters and coronae of Milky Way-like galaxies. In all cases we found monotonically unstable axisymmetric modes. The instability is dominated by the HBI in cool cores and by a combination of TI and MTI in galactic coronae (with the exception of models with very weak poloidal component of the magnetic field, in which the MRI is the dominant instability).

3. For the same galaxy and galaxy-cluster models, we have computed the linear evolution of several nonaxisymmetric disturbances, finding that the linear non-axisymmetric modes behave similarly to axisymmetric modes with the same wave-length and orientation in the meridional plane, so these systems are locally unstable against general perturbations. In particular, in contrast with the unmagnetized case, differential rotation does not stabilize blob-like disturbances in the presence of an ordered magnetic field.

4. Overall, magnetized systems are more prone to local TI than unmagnetized systems: in particular, thermal perturbations tend to be effectively damped by isotropic heat conduction in unmagnetized systems, while, under certain conditions, they can grow monotonically and lead to local condensations in the presence of magnetic fields.

5. Differential rotation plays a crucial role in the studied instabilities. Remarkably, if the magnetic field is sufficiently weak the MRI is dominant even in pressure-supported systems such as galactic coronae and cool cores of galaxy clusters. But also when the MRI is not strong, differential rotation can favor the onset of either the MTI or the HBI, which, combined with the TI, can lead to local condensation of cold gas. The presence of vertical velocity gradient is destabilizing, so baroclinic models tend to be more unstable than barotropic models.

The original motivation of the present investigation was the question of whether cold clouds can form spontaneously throughout the virial-temperature atmospheres of galaxies and clusters of galaxies. In this work we have focused on differentially rotating plasmas, because rotation is potentially relevant in the atmospheres of both galaxies (Marinacci et al. 2011) and galaxy clusters (Bianconi et al. 2013). A necessary condition for the spontaneous formation of cold clouds via TI is that, at least in the linear regime, thermal perturbations grow monotonically. Our results suggest that in the presence of a weak ordered magnetic field, provided that the MRI is not dominant and that the gas temperature is relatively low, thermal perturbations can lead to local condensation through a combination of the TI with either the MTI or the HBI. Therefore the formation of cold clouds via local TI is hampered in the cluster cool cores, while it is possible under specific conditions in galactic coronae. While the gas temperature of galactic and galaxy-cluster atmospheres is relatively well constrained (either observationally or theoretically), much less is known about the distribution of their specific angular momentum and the geometry of their magnetic field. In the hypothesis that the conditions are such that linear thermal perturbations grow monotonically, we are left with the question of the non-linear evolution of these unstable systems, which could be addressed with MHD simulations (see, for the nonrotating case, Kunz et al. 2012, McCourt et al. 2012 and Wagh et al. 2014). One possibility is that, in the non-linear regime, finite-size cold clouds form and the medium becomes multiphase, but it is also possible that the main outcome of the instability is that the magnetic field is rearranged in a configuration that counteracts the development of further instabilities or that the gas becomes highly turbulent and the magnetic field highly tangled.

A limitation of the present work is that, even in the presence of ordered magnetic fields, we have assumed for simplicity pressure to be isotropic, neglecting the Braginskii (1965) viscosity. In fact, in a magnetized plasma anisotropic momentum transport can affect the stability properties of the plasma: for instance, studying non-rotating models of cluster cool cores, Latter \& Kunz (2012) and Kunz et al. (2012) concluded that, in the presence of Braginskii (1965) viscosity, the HBI is substantially reduced, being localized only in the inner $20 \%$ of the cluster core. The question of whether and how much the results of the present work are affected by Braginskii (1965) viscosity can be addressed by 
extending the calculations to the case in which anisotropic pressure is self-consistently included: such an investigation would represent a natural follow-up of this paper.

We are grateful to Steven Balbus for useful discussions. We acknowledge financial support from PRIN MIUR 2010-2011, project "The Chemical and Dynamical Evolution of the Milky Way and Local Group Galaxies", prot. 2010LY5N2T.

\section{APPENDIX}

LIST OF DEFINITIONS

Here is a list of definitions of quantities used in Section 4:

$$
\begin{aligned}
& X_{R R} \equiv \frac{2 \tilde{k}_{R}}{\tilde{k}^{2}}\left(\Gamma_{\Omega R}-\tilde{k}_{z}^{2}\right), \\
& X_{R z} \equiv \frac{2 \tilde{k}_{R}}{\tilde{k}^{2}}\left[\Gamma_{\Omega z}-\frac{\tilde{k}_{z}}{\tilde{k}_{R}}\left(\tilde{k}_{z}^{2}+1\right)\right], \\
& X_{R \rho} \equiv \tilde{c}_{0}^{2} \Gamma_{p R}\left(\frac{\tilde{k}_{z}^{2}+1}{\tilde{k}^{2}}-\frac{\Gamma_{p z}}{\Gamma_{p R}} \frac{\tilde{k}_{R}}{\tilde{k}^{2}}\right), \\
& X_{z R} \equiv \frac{2 \tilde{k}_{z}}{\tilde{k}^{2}}\left(\Gamma_{\Omega R}+\tilde{k}_{R}^{2}+1\right) \text {, } \\
& X_{z z} \equiv \frac{2 \tilde{k}_{z}}{\tilde{k}^{2}}\left(\Gamma_{\Omega z}+\tilde{k}_{z} \tilde{k}_{R}\right) \text {, } \\
& X_{z \rho} \equiv \tilde{c}_{0}^{2} \Gamma_{p z}\left(\frac{\tilde{k}_{R}^{2}+1}{\tilde{k}^{2}}-\frac{\Gamma_{p R}}{\Gamma_{p z}} \frac{\tilde{k}_{R} \tilde{k}_{z}}{\tilde{k}^{2}}\right), \\
& X_{\rho R} \equiv \frac{1}{\gamma}\left(\Gamma_{p R}-\gamma \Gamma_{\rho R}\right), \\
& X_{\rho z} \equiv \frac{1}{\gamma}\left(\Gamma_{p z}-\gamma \Gamma_{\rho z}\right), \\
& X_{\rho \rho} \equiv-\left(\tilde{\omega}_{\mathrm{c}, 0} K^{2}+\tilde{\omega}_{\mathrm{th}}\right) \text {, } \\
& Y_{\rho R} \equiv \frac{\beta \tilde{\omega}_{\mathrm{c}, 0}}{2 \tilde{c}_{0}^{2}}\left[\Gamma_{T R}-2\left(\Gamma_{T R} b_{0 R}+\Gamma_{T z} b_{0 z}\right) b_{0 R}\right] \text {, } \\
& Y_{\rho z} \equiv \frac{\beta \tilde{\omega}_{\mathrm{c}, 0}}{2 \tilde{c}_{0}^{2}}\left[\Gamma_{T z}-2\left(\Gamma_{T R} b_{0 R}+\Gamma_{T z} b_{0 z}\right) b_{0 z}\right], \\
& \dot{X}_{R R} \equiv \frac{\mathrm{d} X_{R R}}{\mathrm{~d} \tau}=\frac{2}{\tilde{k}^{4}}\left[\Gamma_{\Omega R} \tilde{k}_{z}^{4}-\Gamma_{\Omega R}\left(\tilde{k}_{R}^{2}+\Gamma_{\Omega R}-1\right) \tilde{k}_{z}^{2}+2 \Gamma_{\Omega z}\left(\tilde{k}_{R}+\Gamma_{\Omega R}+1\right) \tilde{k}_{R} \tilde{k}_{z}+\Gamma_{\Omega R}^{2}\left(k_{R}^{2}-1\right)\right], \\
& \dot{X}_{R z} \equiv \frac{\mathrm{d} X_{R z}}{\mathrm{~d} \tau}=\frac{2}{\tilde{k}^{4}}\left[\Gamma_{\Omega z} \tilde{k}_{z}^{4}-2 \Gamma_{\Omega R} \tilde{k}_{R} \tilde{k}_{z}^{3}+\Gamma_{\Omega z}\left(3 \tilde{k}_{R}^{2}+2-\Gamma_{\Omega R}\right) \tilde{k}_{z}^{2}\right. \\
& \left.+2\left(\Gamma_{\Omega z}^{2}-\Gamma_{\Omega R}\right) \tilde{k}_{R} \tilde{k}_{z}+\Gamma_{\Omega z}\left(\Gamma_{\Omega R}+1\right) \tilde{k}_{R}^{2}+\Gamma_{\Omega z}\left(1-\Gamma_{\Omega R}\right)\right] \\
& \dot{X}_{R \rho} \equiv \frac{\mathrm{d} X_{R \rho}}{\mathrm{d} \tau}=\frac{\tilde{c}_{0}^{2} \Gamma_{p R}}{\tilde{k}^{4}}\left[2 \tilde{k}_{R}\left(\Gamma_{\Omega R} \tilde{k}_{z}^{2}-\Gamma_{\Omega z} \tilde{k}_{R} \tilde{k}_{z}+\Gamma_{\Omega R}\right)\right. \\
& \left.+\frac{\Gamma_{p z}}{\Gamma_{p R}}\left[\Gamma_{\Omega R} \tilde{k}_{z}^{3}-\Gamma_{\Omega z} \tilde{k}_{R} \tilde{k}_{z}^{2}+\left(1-\tilde{k}_{R}^{2}\right) \Gamma_{\Omega R} \tilde{k}_{z}+\Gamma_{\Omega z} \tilde{k}_{R}\left(\tilde{k}_{R}^{2}+1\right)\right]\right], \\
& \dot{X}_{z R} \equiv \frac{\mathrm{d} X_{z R}}{\mathrm{~d} \tau}=\frac{2}{\tilde{k}^{4}}\left[-2 \Gamma_{\Omega R} \tilde{k}_{R} \tilde{k}_{z}^{3}+\Gamma_{\Omega z}\left(\tilde{k}_{R}^{2}+\Gamma_{\Omega R}+1\right) \tilde{k}_{z}^{2}+2 \Gamma_{\Omega R}^{2} \tilde{k}_{R} \tilde{k}_{z}-\Gamma_{\Omega z}\left(\tilde{k}_{R}^{2}+1\right)\left(\tilde{k}_{R}^{2}+\Gamma_{\Omega R}+1\right)\right] \text {, } \\
& \dot{X}_{z z} \equiv \frac{\mathrm{d} X_{z z}}{\mathrm{~d} \tau}=\frac{2}{\tilde{k}^{4}}\left[-\Gamma_{\Omega R} \tilde{k}_{z}^{4}+\left(\Gamma_{\Omega R} \tilde{k}_{R}^{2}+\Gamma_{\Omega z}^{2}-\Gamma_{\Omega R}\right) \tilde{k}_{z}^{2}-2 \Gamma_{\Omega z} \tilde{k}_{R}\left(\tilde{k}_{R}^{2}-\Gamma_{\Omega R}+1\right) \tilde{k}_{z}-\Gamma_{\Omega z}^{2}\left(\tilde{k}_{R}^{2}+1\right)\right] \text {, } \\
& \dot{X}_{z \rho} \equiv \frac{\mathrm{d} X_{z \rho}}{\mathrm{d} \tau}=\frac{\tilde{c}_{0}^{2} \Gamma_{p z}}{\tilde{k}^{4}}\left[-2 \tilde{k}_{z}\left(\Gamma_{\Omega R} \tilde{k}_{z} \tilde{k}_{R}-\Gamma_{\Omega z} \tilde{k}_{R}^{2}-\Gamma_{\Omega z}\right)\right. \\
& \left.+\frac{\Gamma_{p R}}{\Gamma_{p z}}\left[\Gamma_{\Omega R} \tilde{k}_{z}^{3}-\Gamma_{\Omega z} \tilde{k}_{R} \tilde{k}_{z}^{2}+\left(1-\tilde{k}_{R}^{2}\right) \Gamma_{\Omega R} \tilde{k}_{z}+\Gamma_{\Omega z} \tilde{k}_{R}\left(\tilde{k}_{R}^{2}+1\right)\right]\right] .
\end{aligned}
$$




\section{REFERENCES}

Balbus, S. A. 1991, ApJ, 372, 25

Balbus, S. A. 2000, ApJ, 534, 420

Balbus, S. A., \& Hawley, J. F. 1991, ApJ, 376, 214

Balbus, S. A., \& Hawley, J. F. 1992, ApJ, 400, 610

Balbus, S. A., \& Reynolds, C. S. 2010, ApJ, 720, L97

Balbus, S. A., \& Soker, N. 1989, ApJ, 341, 611

Bertin, G., Lin, C. C., Lowe, S. A., \& Thurstans, R. P. 1989, ApJ, 338, 104

Bianconi, M., Ettori, S., \& Nipoti, C. 2013, MNRAS, 434, 1565

Binney, J., \& Cowie, L. L. 1981, ApJ, 247, 464

Binney, J., Nipoti, C., \& Fraternali, F. 2009, MNRAS, 397, 1804

Braginskii, S. I. 1965, Reviews of Plasma Physics, 1, 205

Chandrasekhar, S. 1960, Proceedings of the National Academy of Science, 46, 253

Cowling, T. G. 1951, ApJ, 114, 272

Dai, X., Anderson, M. E., Bregman, J. N., \& Miller, J. M. 2012, ApJ, 755, 107

Das, U., \& Sharma, P. 2013, MNRAS, 435, 2431

Defouw, R. J. 1970, ApJ, 160, 659

Ferraro, V. C. A. 1937, MNRAS, 97, 458

Field, G. B. 1965, ApJ, 142, 531

Goldreich, P., \& Lynden-Bell, D. 1965, MNRAS, 130, 125

Joung, M. R., Bryan, G. L., \& Putman, M. E. 2012, ApJ, 745, 148

Kunz, M. W. 2011, MNRAS, 417, 602

Kunz, M. W., Bogdanović, T., Reynolds, C. S., \& Stone, J. M. 2012, ApJ, 754, 122
Latter, H. N., \& Kunz, M. W. 2012, MNRAS, 423, 1964

Li, J., Ostriker, J., \& Sunyaev, R. 2013, ApJ, 767, 105

Lin, C. C., \& Shu, F. H. 1964, ApJ, 140, 646

Loewenstein, M. 1990, ApJ, 349, 471

Malagoli, A., Rosner, R., \& Bodo, G. 1987, ApJ, 319, 632

Maller, A. H., \& Bullock, J. S. 2004, MNRAS, 355, 694

Marinacci, F., Fraternali, F., Nipoti, C., et al. 2011, MNRAS, 415,1534

Mathews, W. G., \& Bregman, J. N. 1978, ApJ, 224, 308

Mathews, W. G., \& Brighenti, F. 2003, ARA\&A, 41, 191

McCourt, M., Sharma, P., Quataert, E., \& Parrish, I. J. 2012, MNRAS, 419, 3319

Miller, M. J., \& Bregman, J. N. 2013, ApJ, 770, 118

Nelson, R. P., Gressel, O., \& Umurhan, O. M. 2013, MNRAS, 435,2610

Nipoti, C. 2010, MNRAS, 406, 247

Nipoti, C., \& Posti, L. 2013, MNRAS, 428, 815

Parrish, I. J., McCourt, M., Quataert, E., \& Sharma, P. 2012, MNRAS, 422, 704

Quataert, E. 2008, ApJ, 673, 758

Rosati, P., Borgani, S., \& Norman, C. 2002, ARA\&A, 40, 539

Spitzer, L. 1962, Physics of Fully Ionized Gases, New York: Interscience (2nd edition), 1962,

Sutherland, R. S., \& Dopita, M. A. 1993, ApJS, 88, 253

Tassoul, J.-L. 1978, Theory of Rotating Stars. Princeton University Press, Princeton

Velikhov, E. P. 1959, Soviet Phys. JETP, 9, 995

Wagh, B., Sharma, P., \& McCourt, M. 2014, MNRAS, 439, 2822 